Supporting Information for the article

\title{
Synthesis and Properties of a Triruthenium Hydrido Complex Capped by a $\mu 3$-Oxoboryl Ligand
}

Takeshi Kaneko, Hayato Ninagawa, Moe Matsuoka, Toshiro Takao*

$\dagger$ Department of Chemical Science and Engineering, School of Materials and Chemical

Technology, Tokyo Institute of Technology, 2-12-1 O-okayama, Meguro-ku, Tokyo 152-8552, Japan

Contents

1. Crystal data and results of XRD studies $\quad \mathrm{S}-2$

2. Results of DFT calculations S-10

3. NMR and IR spectra of compounds S-19 


\section{Crystal data and results of XRD studies}

Table S1. Crystallographic Data for 3, 5, 6, 7, 2a, and $\left[\{\mathrm{Cp} * \mathrm{Ru}(\mu-\mathrm{H})\}_{3}\left(\mu_{3}-\mathrm{AlEt}\right)\right]$

\begin{tabular}{|c|c|c|c|}
\hline & 3 & 5 & 6 \\
\hline \multicolumn{4}{|l|}{ (a) Crystal data } \\
\hline Empirical formula & $\mathrm{C}_{30} \mathrm{H}_{49} \mathrm{BORu}_{3} \cdot \mathrm{C}_{7} \mathrm{H}_{8}$ & $\mathrm{C}_{96} \mathrm{H}_{98} \mathrm{~B}_{4} \mathrm{~F}_{30} \mathrm{O}_{2} \mathrm{Ru}_{6} \cdot \mathrm{C}_{3} \mathrm{H}_{6} \mathrm{O}$ & $\mathrm{C}_{33} \mathrm{H}_{58} \mathrm{BOPRu}_{3}$ \\
\hline Formula weight & 831.84 & 2561.48 & 815.78 \\
\hline Crystal description & Block & Platelet & Block \\
\hline Crystal color & Black & Black & Brown \\
\hline Crystal size (mm) & $0.57 \times 0.40 \times 0.28$ & $0.56 \times 0.16 \times 0.12$ & $0.36 \times 0.19 \times 0.18$ \\
\hline Crystalizing solution & toluene $\left(-20^{\circ} \mathrm{C}\right)$ & acetone $\left(-30^{\circ} \mathrm{C}\right)$ & toluene $\left(-30^{\circ} \mathrm{C}\right)$ \\
\hline Crystal system & Monoclinic & Monoclinic & Triclinic \\
\hline Space group & $P 2_{1} / c(\# 14)$ & $P 2_{1} / c(\# 14)$ & $P-1(\# 2)$ \\
\hline$a(\AA)$ & $24.6640(6)$ & $12.4426(5)$ & $9.7895(4)$ \\
\hline$b(\AA)$ & $14.5035(3)$ & $43.1088(16)$ & $10.5117(4)$ \\
\hline$c(\AA)$ & $22.2803(5)$ & $18.4883(9)$ & $17.4775(7)$ \\
\hline$\alpha\left(^{\circ}\right)$ & & & $89.5820(10)$ \\
\hline$\beta\left(^{\circ}\right)$ & $115.2260(10)$ & $92.0550(10)$ & $89.7350(10)$ \\
\hline$\gamma\left({ }^{\circ}\right)$ & & & $77.5720(10)$ \\
\hline Volume $\left(\AA^{3}\right)$ & $7209.9(3)$ & $9910.5(7)$ & $1756.31(12)$ \\
\hline$Z$ value & 8 & 4 & 2 \\
\hline$D_{\text {calc }}\left(\mathrm{g} / \mathrm{cm}^{3}\right)$ & 1.533 & 1.717 & 1.543 \\
\hline Measurement temp. $\left({ }^{\circ} \mathrm{C}\right)$ & -150 & -113 & -133 \\
\hline$\mu(\mathrm{MoK} \alpha)\left(\mathrm{mm}^{-1}\right)$ & 1.267 & 0.997 & 1.342 \\
\hline \multicolumn{4}{|l|}{ (b) Intensity measurements } \\
\hline Diffractometer & Rigaku R-AXIS RAPID & Rigaku R-AXIS RAPID & Rigaku R-AXIS RAPID \\
\hline Radiation & $\operatorname{MoK} \alpha(\lambda=0.71069 \AA)$ & $\operatorname{MoK} \alpha(\lambda=0.71069 \AA)$ & $\operatorname{MoK} \alpha(\lambda=0.71069 \AA)$ \\
\hline Monochromator & Graphite & Graphite & Graphite \\
\hline $2 \theta \max \left({ }^{\circ}\right)$ & 55 & 55 & 55 \\
\hline Reflections collected & 69957 & 151881 & 17397 \\
\hline Independent reflections & $16450\left(R_{\mathrm{int}}=0.0541\right)$ & $22653\left(R_{\mathrm{int}}=0.0522\right)$ & $7971\left(R_{\mathrm{int}}=0.0281\right)$ \\
\hline Reflections observed $(>2 \sigma)$ & 13717 & 19239 & 7096 \\
\hline Abs. Correction type & Empirical & Numerical & Numerical \\
\hline Abs. Transmission & $\begin{array}{l}0.5245(\min ) \\
1.0000(\max )\end{array}$ & $\begin{array}{l}0.7573(\min ) \\
1.0000(\max )\end{array}$ & $\begin{array}{l}0.7918(\min ) \\
0.8731(\max )\end{array}$ \\
\hline \multicolumn{4}{|c|}{ (c) Refinement (Shelxl-2018/1) } \\
\hline$R_{1}(I>2 \sigma(I))$ & 0.0427 & 0.0305 & 0.0314 \\
\hline$w R_{2}(I>2 \sigma(I))$ & 0.1012 & 0.0646 & 0.0703 \\
\hline$R_{1}$ (all data) & 0.0537 & 0.0399 & 0.0766 \\
\hline$w R_{2}$ (all data) & 0.1073 & 0.0685 & 0.0952 \\
\hline Data/Restraints/Parameters & $16450 / 0 / 784$ & $22653 / 0 / 1343$ & 7971 / 0 / 372 \\
\hline GOF & 1.017 & 1.018 & 1.080 \\
\hline $\begin{array}{l}\text { Largest diff. Peak and hole } \\
\left(\mathrm{e} \AA^{-3}\right)\end{array}$ & 1.770 and -1.855 & 0.858 and -0.714 & 1.390 and -0.803 \\
\hline CCDC deposition number & 1901653 & 1901654 & 1901655 \\
\hline
\end{tabular}


Table S1 (continued). Crystallographic Data for 3, 5, 6, 7, 2a, and $\left[\{\mathrm{Cp} * \mathrm{Ru}(\mu-\mathrm{H})\}_{3}\left(\mu_{3}-\mathrm{AlEt}\right)\right]$

\begin{tabular}{|c|c|c|c|}
\hline & 7 & $2 a$ & $\{\mathrm{Cp} * \mathrm{Ru}(\mu-\mathrm{H})\}_{3}\left(\mu_{3}-\mathrm{AlEt}\right)$ \\
\hline \multicolumn{4}{|l|}{ (a) Crystal data } \\
\hline Empirical formula & $\mathrm{C}_{33} \mathrm{H}_{47} \mathrm{BO}_{4} \mathrm{Ru}_{3}$ & $\mathrm{C}_{30} \mathrm{H}_{49} \mathrm{BRu}_{3}$ & $\mathrm{C}_{32} \mathrm{H}_{53} \mathrm{AlRu}_{3} \cdot \mathrm{C}_{4} \mathrm{H}_{8} \mathrm{O}$ \\
\hline Formula weight & 821.72 & 723.71 & 840.03 \\
\hline Crystal description & Platelet & Block & Block \\
\hline Crystal color & Black & Black & Dark red \\
\hline Crystal size (mm) & $0.15 \times 0.13 \times 0.03$ & $0.54 \times 0.38 \times 0.29$ & $0.70 \times 0.60 \times 0.50$ \\
\hline Crystalizing solution & $\mathrm{CH}_{2} \mathrm{Cl}_{2} /$ pentane $\left(-30^{\circ} \mathrm{C}\right)$ & & $\operatorname{THF}\left(-30^{\circ} \mathrm{C}\right)$ \\
\hline Crystal system & Orthorhombic & Orthorhombic & Orthorhombic \\
\hline Space group & Pbca (\#61) & $\operatorname{Pna2}_{1}(\# 33)$ & Pnma (\#62) \\
\hline$a(\AA)$ & $16.9052(4)$ & $27.7129(7)$ & $14.6340(4)$ \\
\hline$b(\AA)$ & $18.4414(3)$ & $11.6471(3)$ & $16.6511(4)$ \\
\hline$c(\AA)$ & $20.7153(5)$ & $18.6286(4)$ & $14.8269(3)$ \\
\hline \multicolumn{4}{|l|}{$\alpha\left(^{\circ}\right)$} \\
\hline \multicolumn{4}{|l|}{$\beta\left(^{\circ}\right)$} \\
\hline \multicolumn{4}{|l|}{$\gamma\left({ }^{\circ}\right)$} \\
\hline Volume $\left(\AA^{3}\right)$ & $6458.1(2)$ & $6012.8(3)$ & $3612.90(15)$ \\
\hline$Z$ value & 8 & 8 & 4 \\
\hline$D_{\text {calc }}\left(\mathrm{g} / \mathrm{cm}^{3}\right)$ & 1.690 & 1.599 & 1.544 \\
\hline Measurement temp. $\left({ }^{\circ} \mathrm{C}\right)$ & -130 & -130 & -150 \\
\hline$\mu(\operatorname{MoK} \alpha)\left(\mathrm{mm}^{-1}\right)$ & 1.420 & 1.504 & 1.288 \\
\hline \multicolumn{4}{|l|}{ (b) Intensity measurements } \\
\hline Diffractometer & Rigaku R-AXIS RAPID & Rigaku R-AXIS RAPID & Rigaku R-AXIS RAPID \\
\hline Radiation & $\operatorname{MoK} \alpha(\lambda=0.71069 \AA)$ & $\operatorname{MoK} \alpha(\lambda=0.71069 \AA)$ & $\operatorname{MoK} \alpha(\lambda=0.71069 \AA)$ \\
\hline Monochromator & Graphite & Graphite & Graphite \\
\hline $2 \theta \max \left(\left(^{\circ}\right)\right.$ & 55 & 55 & 60 \\
\hline Reflections collected & 63683 & 93321 & 39237 \\
\hline Independent reflections & $7327\left(R_{\mathrm{int}}=0.0366\right)$ & $13538\left(R_{\text {int }}=0.0621\right)$ & $5453\left(R_{\mathrm{int}}=0.0544\right)$ \\
\hline Reflections observed $(>2 \sigma)$ & 6874 & 12746 & 4487 \\
\hline Abs. Correction type & Empirical & Numerical & Empirical \\
\hline Abs. Transmission & $0.7282(\mathrm{~min})$ & $\begin{array}{l}0.5389(\min ) \\
0.7365(\max )\end{array}$ & $0.7641(\min )$ \\
\hline \multicolumn{4}{|c|}{ (c) Refinement (Shelxl-2018/1) } \\
\hline$R_{1}(I>2 \sigma(I))$ & 0.0383 & 0.0392 & 0.0429 \\
\hline$w R_{2}(I>2 \sigma(I))$ & 0.0935 & 0.0906 & 0.1123 \\
\hline$R_{1}$ (all data) & 0.0409 & 0.0424 & 0.0521 \\
\hline$w R_{2}$ (all data) & 0.0952 & 0.0926 & 0.1157 \\
\hline Data/Restraints/Parameters & 7327 / 0 / 393 & 13538 / 4 / 623 & $5453 / 0 / 203$ \\
\hline GOF & 1.061 & 1.047 & 1.090 \\
\hline $\begin{array}{l}\text { Largest diff. Peak and hole } \\
\left(\mathrm{e} . \AA^{-3}\right)\end{array}$ & 6.184 and -0.984 & 1.509 and -0.687 & 3.040 and -1.481 \\
\hline CCDC deposition number & 1901656 & 1901657 & 1901658 \\
\hline
\end{tabular}


(a) $(\mathrm{Cp} * \mathrm{Ru})_{3}\left(\mu_{3}-\mathrm{BO}\right)\left(\mu_{3}-\mathrm{H}\right)(\mu-\mathrm{H})_{3}(3)$

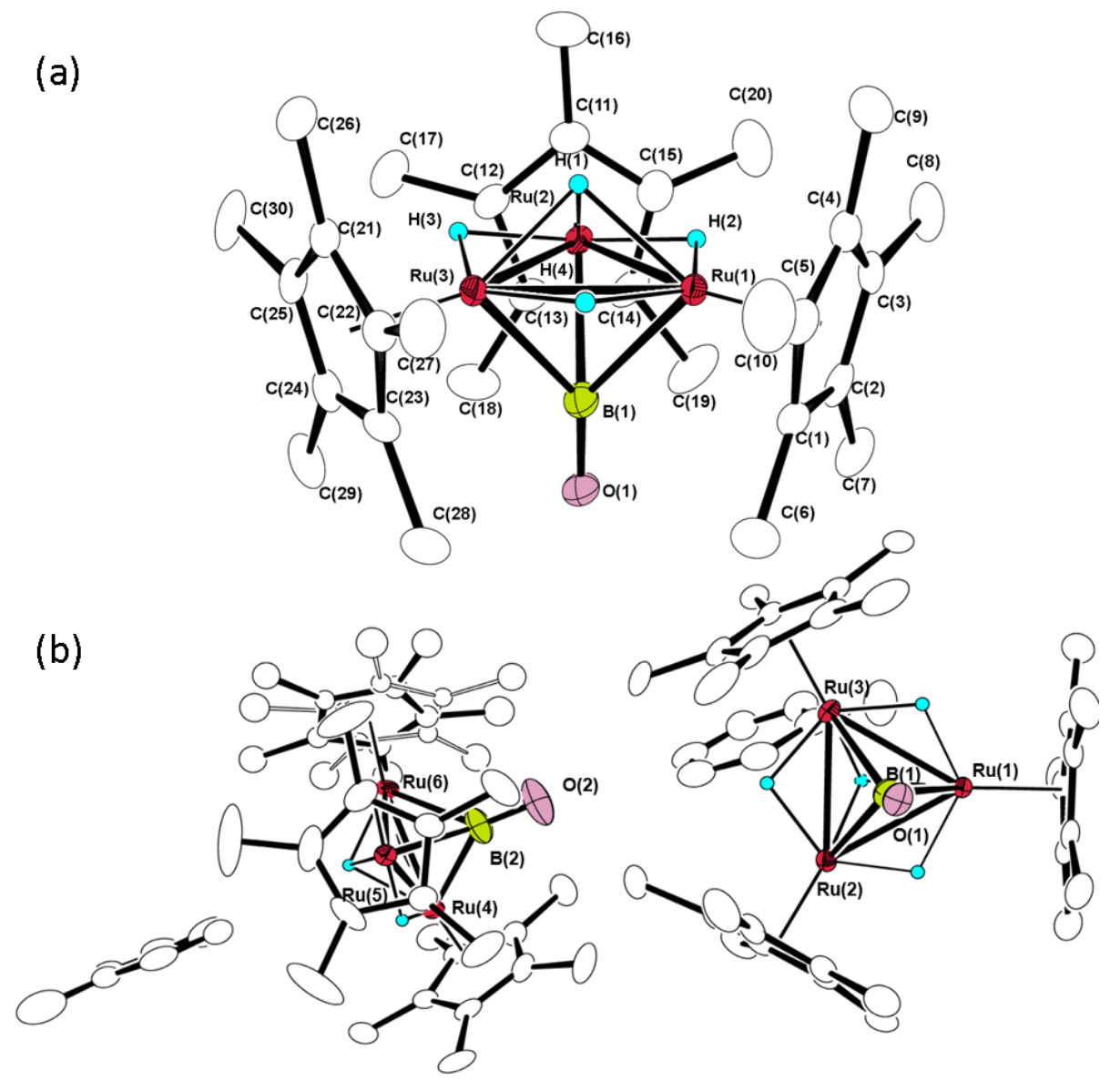

Figure S1. (a) Molecular structure and labeling scheme of $\mathbf{3}$ with thermal ellipsoids at $30 \%$ probability level. (b) Independent molecules of $\mathbf{3}$ and solvent molecule (toluene) in the unit cell.

Table S2. Selected bond lengths $[\AA]$ and angles $\left[^{\circ}\right]$ for $\mathbf{3}$.

Mole A

Ru1 B1 2.302(5)

Ru1 Ru2 2.7113(4)

Ru1 Ru3 2.7170(4)

Ru1 H1 1.97(6)

Ru1 H2 1.74(5)

Ru1 H4 1.67(4)

Ru2 B1 2.320(6)

Ru2 Ru3 2.7205(5)

Ru2 H1 1.94(6)

Ru2 H2 1.65(5)

Ru2 H3 1.75(6)

Ru3 B1 2.305(5)

Ru3 H1 1.91(7)

Ru3 H3 1.71(6)

Ru3 H4 1.65(4)

B1 O1 1.231(6)
B1 Ru1 Ru2 54.39(14)

B1 Ru1 Ru3 53.91(13)

Ru2 Ru1 Ru3 60.155(11)

B1 Ru2 Ru1 53.77(13)

B1 Ru2 Ru3 53.71(13)

Ru1 Ru2 Ru3 60.027(11)

B1 Ru3 Ru1 53.80(13)

B1 Ru3 Ru2 54.22(15)

Ru1 Ru3 Ru2 59.818(11)

O1 B1 Ru1 137.5(4)

O1 B1 Ru3 137.6(4)

Ru1 B1 Ru3 72.29(16)

O1 B1 Ru2 136.5(4)

Ru1 B1 Ru2 71.85(16)

Ru3 B1 Ru2 72.07(16)

\section{Mole B}

Ru4 B2 2.190(7)

B2 Ru4 Ru6 53.6(2)

Ru4 Ru6 2.7133(5)

B2 Ru4 Ru5 55.1(2)

Ru6 Ru4 Ru5 59.814(12)

B2 Ru5 Ru6 52.6(2)

B2 Ru5 Ru4 50.67(19)

Ru6 Ru5 Ru4 59.896(12)

B2 Ru6 Ru5 54.81(19)

O2 B2 Ru4 138.2(7)

O2 B2 Ru6 133.4(7)

Ru4 B2 Ru6 75.2(2)

O2 B2 Ru5 136.2(5)

Ru4 B2 Ru5 74.2(2)

Ru6 B2 Ru5 72.6(2) 
(b) $(\mathrm{Cp} * \mathrm{Ru})_{3}\left\{\mu-\mathrm{BO} \cdots \mathrm{B}\left(\mathrm{C}_{6} \mathrm{~F}_{5}\right)_{3}\right\}\left(\mu_{3}-\mathrm{H}\right)(\mu-\mathrm{H})_{3}(\mathbf{5})$

(a)

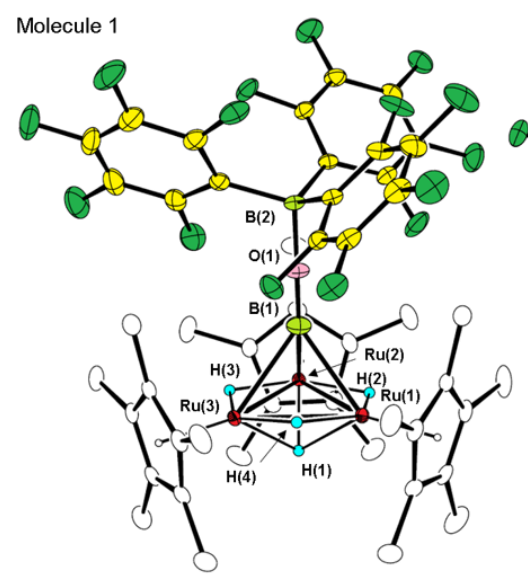

Molecule 2

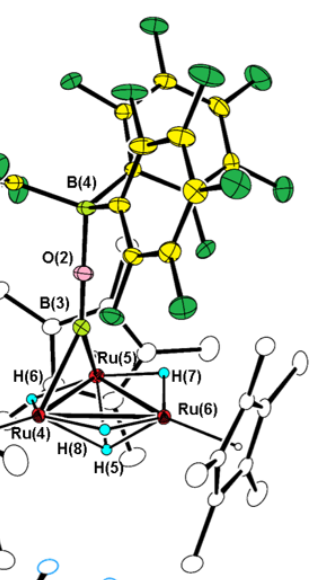

(b)

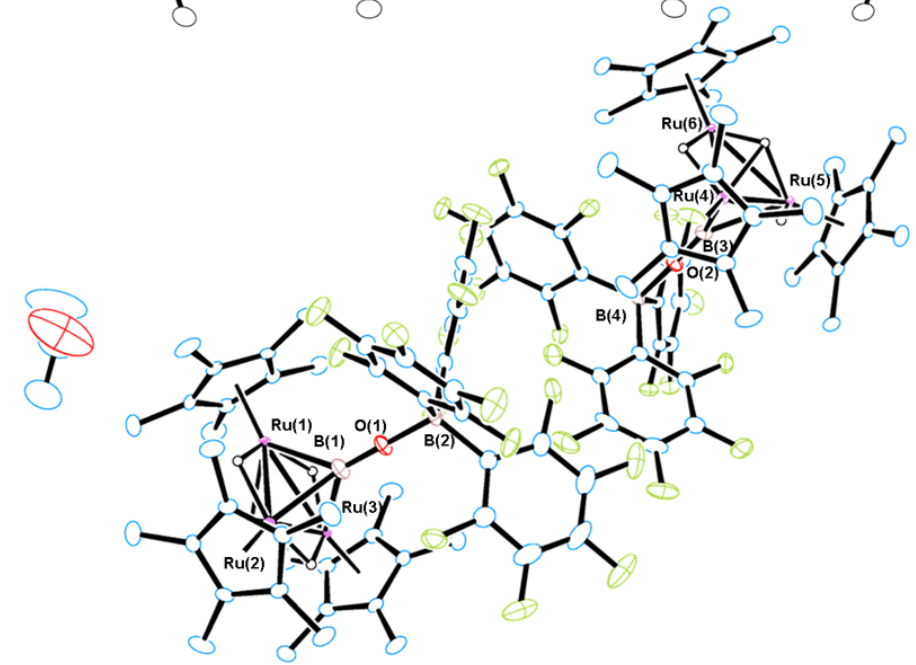

Figure S2. (a) Molecular structures and labeling schemes of $\mathbf{5}$ with thermal ellipsoids at $30 \%$ probability level. (b) Independent molecules of $\mathbf{5}$ and solvent molecule (acetone) in the unit cell.

Table S3. Selected bond lengths $[\AA]$ and angles $\left[{ }^{\circ}\right]$ for 3 .

$\begin{array}{llll}\text { Molecule 1 } & \text { Ru3 H1 1.88(4) } & \text { Ru3 B1 Ru2 71.18(10) } & \text { Ru5 H6 1.66(3) } \\ \text { Ru1 B1 2.395(4) } & \text { Ru3 H3 1.71(3) } & \text { B1 O1 B2 178.9(3) } & \text { Ru5 H7 1.69(3) } \\ \text { Ru1 Ru2 2.7041(3) } & \text { Ru3 H4 1.66(3) } & & \text { Ru6 H5 1.80(4) } \\ \text { Ru1 Ru3 2.7402(3) } & & \text { Molecule 2 } & \text { Ru6 H7 1.68(3) } \\ \text { Ru2 B1 2.196(4) } & \text { B1 Ru1 Ru2 50.57(9) } & \text { Ru4 B3 2.144(3) } & \text { Ru6 H8 1.65(3) } \\ \text { Ru2 Ru3 2.7119(3) } & \text { B1 Ru1 Ru3 56.52(9) } & \text { Ru4 Ru5 2.6886(3) } & \\ \text { Ru3 B1 2.450(4) } & \text { Ru2 Ru1 Ru3 59.748(7) } & \text { Ru4 Ru6 2.7364(3) } & \text { B3 Ru4 Ru5 55.92(9) } \\ \text { B1 O1 1.260(4) } & \text { B1 Ru2 Ru1 57.41(10) } & \text { Ru5 B3 2.316(3) } & \text { B3 Ru4 Ru6 64.86(8) } \\ \text { O1 B2 1.511(3) } & \text { B1 Ru2 Ru3 58.79(10) } & \text { Ru5 Ru6 2.7479(3) } & \text { Ru5 Ru4 Ru6 60.858(7) } \\ \text { B2 C37 1.645(4) } & \text { Ru1 Ru2 Ru3 60.788(7) } & \text { B3 O2 1.259(4) } & \text { B3 Ru5 Ru4 50.06(8) } \\ \text { B2 C43 1.653(4) } & \text { B1 Ru3 Ru1 54.62(9) } & \text { O2 B4 1.509(3) } & \text { B3 Ru5 Ru6 62.81(8) } \\ \text { B2 C31 1.656(4) } & \text { B1 Ru3 Ru2 50.03(9) } & \text { B4 C79 1.645(4) } & \text { Ru4 Ru5 Ru6 60.432(7) } \\ \text { Ru1 H1 1.90(4) } & \text { Ru1 Ru3 Ru2 59.464(7) } & \text { B4 C91 1.646(4) } \\ \text { Ru1 H2 1.67(3) } & \text { O1 B1 Ru1 135.6(3) } & \text { B4 C85 1.654(4) } & \text { Ru4 Ru6 Ru5 58.711(8) } \\ \text { Ru1 H4 1.70(3) } & \text { O1 B1 Ru3 133.4(3) } & \text { Ru4 H5 2.01(4) } & \text { O2 B3 Ru4 144.6(2) } \\ \text { Ru2 H1 1.91(4) } & \text { Ru1 B1 Ru3 68.86(9) } & \text { Ru4 H6 1.65(3) } & \text { O2 B3 Ru5 138.3(2) } \\ \text { Ru2 H2 1.70(3) } & \text { O1 B1 Ru2 144.3(3) } & \text { Ru4 H8 1.74(3) } & \text { Ru4 B3 Ru5 74.02(10) } \\ \text { Ru2 H3 1.66(3) } & \text { Ru1 B1 Ru2 72.02(10) } & \text { Ru5 H5 1.98(4) } & \text { B3 O2 B4 177.7(3) } \\ & & \end{array}$


(c) $(\mathrm{Cp} * \mathrm{Ru})_{3}(\mu-\mathrm{BO})(\mu-\mathrm{H})_{3}\left(\mu_{3}-\mathrm{H}\right)\left(\mathrm{PMe}_{3}\right)(\mathbf{6})$

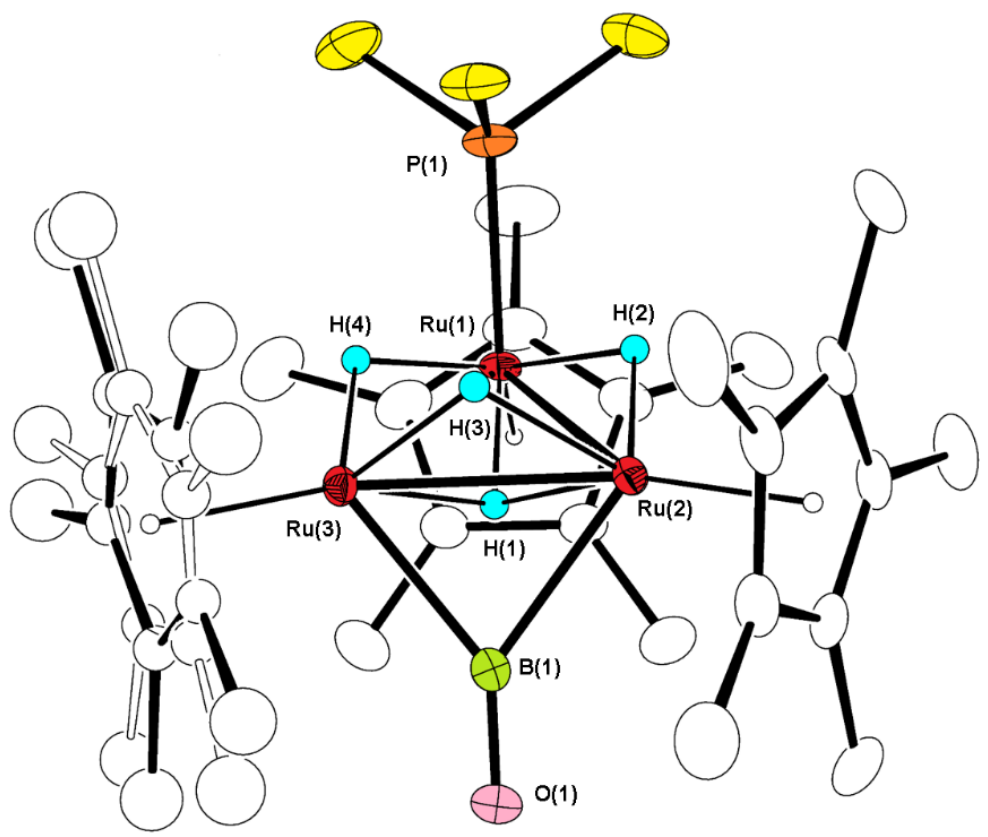

Figure S3. Molecular structure and labeling scheme of $\mathbf{6}$ with thermal ellipsoids at 30\% probability level.

Table S4. Selected bond lengths $[\AA]$ and angles $\left[{ }^{\circ}\right]$ for $\mathbf{6}$.

$\begin{array}{ll}\text { Ru1 P1 2.2884(9) } & \text { Ru1 H1 1.72(5) } \\ \text { Ru1 Ru2 2.9953(3) } & \text { Ru1 H2 1.70(4) } \\ \text { Ru1 Ru3 3.0002(4) } & \text { Ru1 H4 1.67(4) } \\ \text { Ru2 B1 2.174(4) } & \text { Ru2 H1 1.93(5) } \\ \text { Ru2 Ru3 2.6459(3) } & \text { Ru2 H2 1.86(5) } \\ \text { Ru3 B1 2.164(4) } & \text { Ru2 H3 1.72(4) } \\ \text { P1 C3 1.822(4) } & \text { Ru3 H1 1.90(5) } \\ \text { P1 C1 1.828(4) } & \text { Ru3 H3 1.69(4) } \\ \text { P1 C2 1.829(4) } & \text { Ru3 H4 1.86(4) }\end{array}$

O1 B1 1.230(5)

\author{
P1 Ru1 Ru2 94.50(3) \\ P1 Ru1 Ru3 95.17(3) \\ Ru2 Ru1 Ru3 52.375(8) \\ B1 Ru2 Ru3 52.24(10) \\ B1 Ru2 Ru1 90.86(11) \\ Ru3 Ru2 Ru1 63.908(9) \\ B1 Ru3 Ru2 52.59(10) \\ B1 Ru3 Ru1 90.93(10) \\ Ru1 Ru3 Ru2 63.72(1) \\ C3 P1 C1 100.4(2)
}

\begin{abstract}
C3 P1 C2 100.5(2)
1 P1 C2 100.6(2)

C3 P1 Ru1 120.02(12)

C1 P1 Ru1 116.13(16)

C2 P1 Ru1 116.03(14)

O1 B1 Ru3 142.9(3)

O1 B1 Ru2 141.8(3)

Ru3 B1 Ru2 75.17(13)
\end{abstract}


(d) $\{\mathrm{Cp} * \mathrm{Ru}(\mathrm{CO})\}_{3}(\mu-\mathrm{BO})(\mu-\mathrm{H})_{2}(7)$

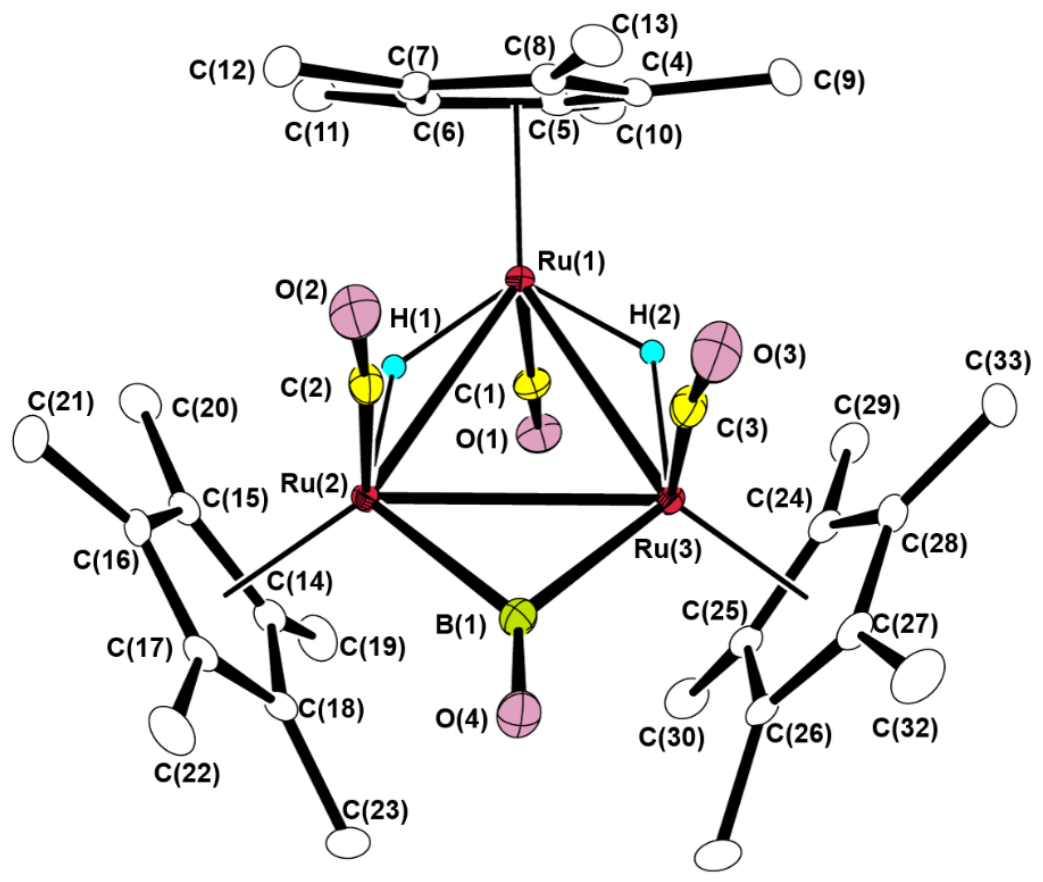

Figure S4. Molecular structure and labeling scheme of $\mathbf{7}$ with thermal ellipsoids at $30 \%$ probability level.

Table S5. Selected bond lengths $[\AA]$ and angles $\left[{ }^{\circ}\right]$ for 7 .

$\begin{array}{lll}\text { Ru1 C1 1.845(4) } & \text { Ru3 B1 2.174(5) } & \text { C2 Ru2 B1 80.19(17) } \\ \text { Ru1 Ru2 3.0140(4) } & \text { Ru3 H2 1.70(6) } & \text { C2 Ru2 Ru3 87.97(12) } \\ \text { Ru1 Ru3 3.0167(4) } & \text { B1 O4 1.236(5) } & \text { B1 Ru2 Ru3 47.09(12) } \\ \text { Ru1 H1 1.62(6) } & \text { C1 O1 1.152(5) } & \text { C2 Ru2 Ru1 86.78(12) } \\ \text { Ru1 H2 1.62(6) } & \text { C2 O2 1.143(5) } & \text { B1 Ru2 Ru1 106.61(12) } \\ \text { Ru2 C2 1.850(4) } & \text { C3 O3 1.143(5) } & \text { Ru3 Ru2 Ru1 60.675(9) } \\ \text { Ru2 B1 2.194(4) } & & \text { C3 Ru3 B1 78.60(17) } \\ \text { Ru2 Ru3 2.9578(4) } & \text { C1 Ru1 Ru2 91.28(12) } & \text { C3 Ru3 Ru2 93.14(12) } \\ \text { Ru2 H1 1.63(6) } & \text { C1 Ru1 Ru3 87.38(11) } & \text { B1 Ru3 Ru2 47.66(11) } \\ \text { Ru3 C3 1.853(4) } & \text { Ru2 Ru1 Ru3 58.741(9) } & \text { C3 Ru3 Ru1 95.42(12) }\end{array}$

B1 Ru3 Ru1 107.08(11) Ru2 Ru3 Ru1 60.585(9) O4 B1 Ru3 136.9(3) O4 B1 Ru2 137.9(3) Ru3 B1 Ru2 85.26(16) O1 C1 Ru1 173.0(3) O2 C2 Ru2 174.6(4) O3 C3 Ru3 172.2(4) 
(e) $\{\mathrm{Cp} * \mathrm{Ru}(\mu-\mathrm{H})\}_{3}\left(\mu_{3}-\mathrm{BH}\right)$

(a)

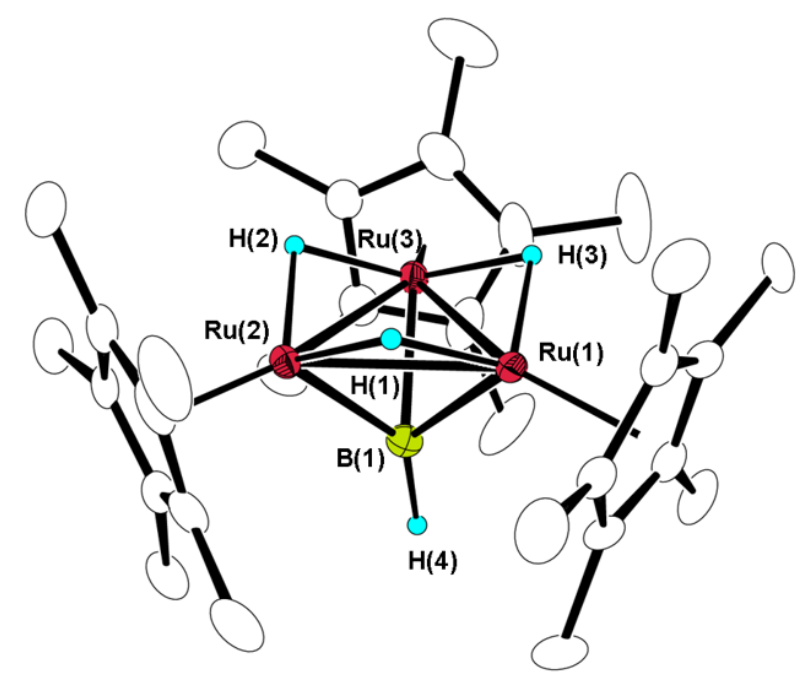

(b)

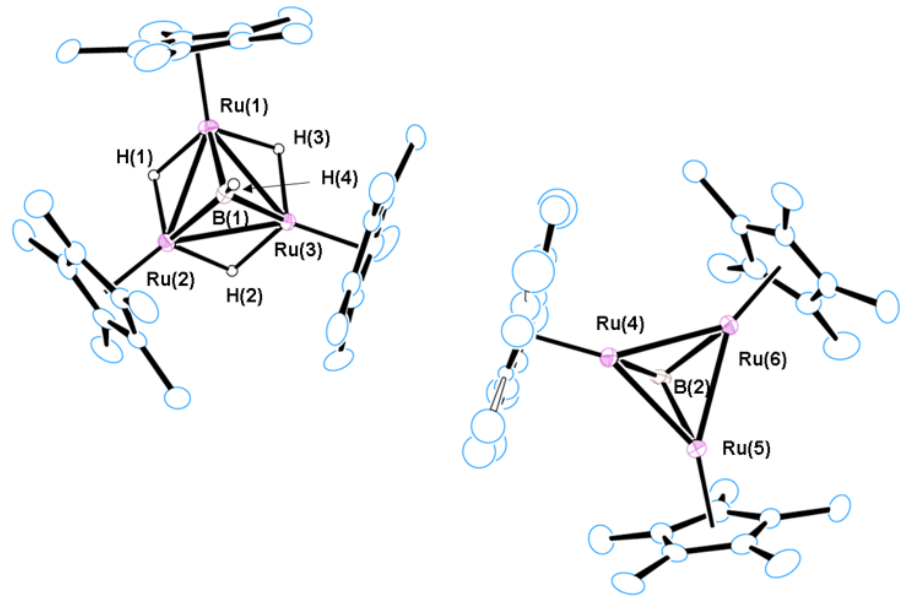

Figure S5. (a) Molecular structure and labeling scheme of $\left[\{\mathrm{Cp} * \mathrm{Ru}(\mu-\mathrm{H})\}_{3}\left(\mu_{3}-\mathrm{BH}\right)\right](\mathbf{2 a})$ with thermal ellipsoids at the $30 \%$ probability level. (b) Independent molecules of $\mathbf{2 a}$ in the unit cell.

Table S6. Selected bond lengths $[\AA]$ and angles $\left[^{\circ}\right]$ for $\left[\{\mathrm{Cp} * \mathrm{Ru}(\mu-\mathrm{H})\}_{3}\left(\mu_{3}-\mathrm{BH}\right)\right](\mathbf{2 a})$.

\section{Molecule 1}

Ru1 B1 2.120(9)

Ru1 Ru2 2.6529(8)

Ru1 Ru3 2.6538(8)

Ru1 H1 1.68(9)

Ru1 H3 1.77(12)

Ru2 B1 2.108(10)

Ru2 Ru3 2.6539(8)

Ru2 H1 1.68(9)

Ru2 H2 1.75(11)

Ru3 B1 2.120(8)

Ru3 H2 1.74(11)

Ru3 H3 1.77(12)
B1 H4 1.24(15)

B1 Ru1 Ru2 50.9(3)

B1 Ru1 Ru3 51.3(2)

Ru2 Ru1 Ru3 60.01(2)

B1 Ru2 Ru1 51.3(3)

B1 Ru2 Ru3 51.3(2)

Ru1 Ru2 Ru3 60.01(2)

B1 Ru3 Ru1 51.2(2)

B1 Ru3 Ru2 50.9(3)

Ru1 Ru3 Ru2 59.98(2)

Ru2 B1 Ru1 77.7(3)

Ru2 B1 Ru3 77.8(3)
Ru3 B1 Ru1 77.5(3)

Ru2 B1 H4 140(8)

Ru3 B1 H4 131(7)

Ru1 B1 H4 129(8)

Molecule 2

Ru4 B2 2.107(10)

Ru4 Ru5 2.6609(8)

Ru4 Ru6 2.6521(8)

Ru5 B2 2.129(9)

Ru5 Ru6 2.6675(9)

Ru6 B2 2.149(10)
B2 Ru4 Ru5 51.5(3)

B2 Ru4 Ru6 52.2(3)

Ru5 Ru4 Ru6 60.3(2)

B2 Ru5 Ru4 50.7(3)

B2 Ru5 Ru6 51.8(3)

Ru4 Ru5 Ru6 59.70(2)

B2 Ru6 Ru4 50.7(3)

B2 Ru6 Ru5 51.1(2)

Ru4 Ru6 Ru5 60.03(2)

Ru4 B2 Ru5 77.8(3)

Ru4 B2 Ru6 77.1(4)

Ru5 B2 Ru6 77.2(3) 
(f) $\{\mathrm{Cp} * \mathrm{Ru}(\mu-\mathrm{H})\}_{3}\left(\mu_{3}-\mathrm{AlEt}\right)$

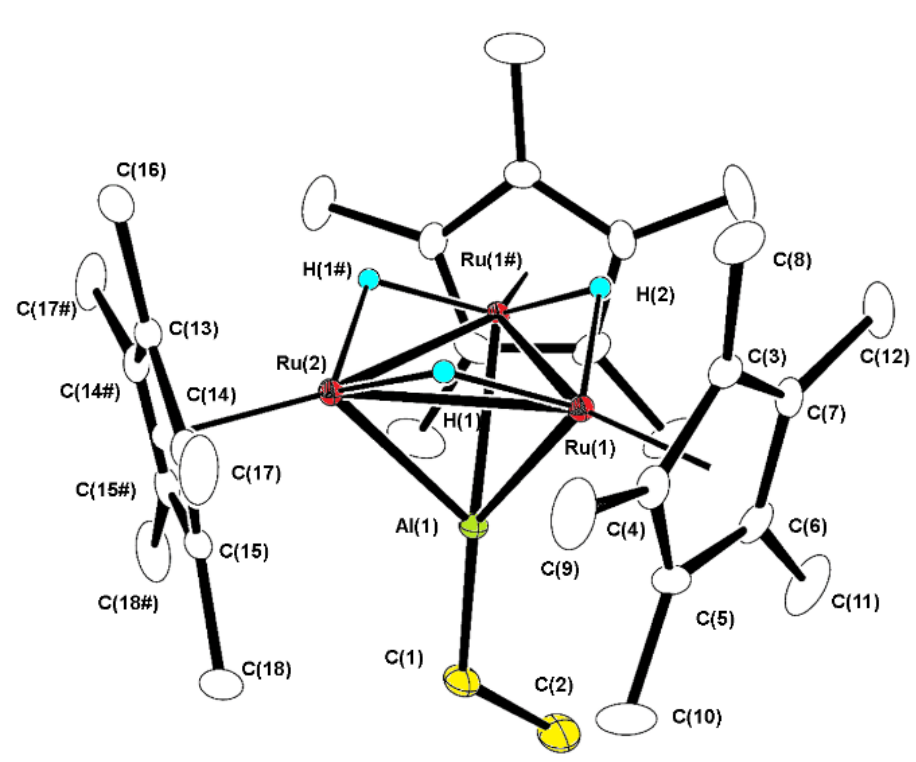

Figure S6. Molecular structure and labeling scheme of $\left[\{\mathrm{Cp} * \mathrm{Ru}(\mu-\mathrm{H})\}_{3}\left(\mu_{3}-\mathrm{AlEt}\right)\right]$ with thermal ellipsoids at the $30 \%$ probability level. A solvent molecule (THF) was omitted for clarity.

Table S7. Selected bond lengths $[\AA]$ and angles $\left[^{\circ}\right]$ for $\{\mathrm{Cp} * \mathrm{Ru}(\mu-\mathrm{H})\}_{3}\left(\mu_{3}-\mathrm{AlEt}\right)$.

$\begin{array}{llll}\text { Ru1 Al1 2.4792(11) } & \text { Ru2 H1 1.74(4) } & \text { Ru1 Ru2 Ru1\# 59.763(12) } & \text { Ru3 Ru2 Ru1 60.675(9) } \\ \text { Ru1 Ru2 2.7383(4) } & \text { Al1 C1 1.981(5) } & \text { Ru1 Ru2 A11 56.42(3) } & \text { A11 C1 C2 123.6(4) } \\ \text { Ru1 Ru1(\#) 2.7285(5) } & \text { Ru2 H1 1.63(6) } & \text { Ru1 A11 Ru1\# 66.77(4) } & \\ \text { Ru1 H1 1.65(4) } & & \text { Ru1 A11 Ru2 66.95(3) } \\ \text { Ru1 H2 1.72(4) } & \text { Ru1\# Ru1 Ru2 60.118(6) } & \text { Ru1 A11 C1 142.98(7) } \\ \text { Ru2 Al1 2.4855(14) } & \text { Ru1\# Ru1 Al1 56.615(18) } & \text { Ru2 A11 C1 134.99(18) }\end{array}$




\section{Results of DFT calculations}

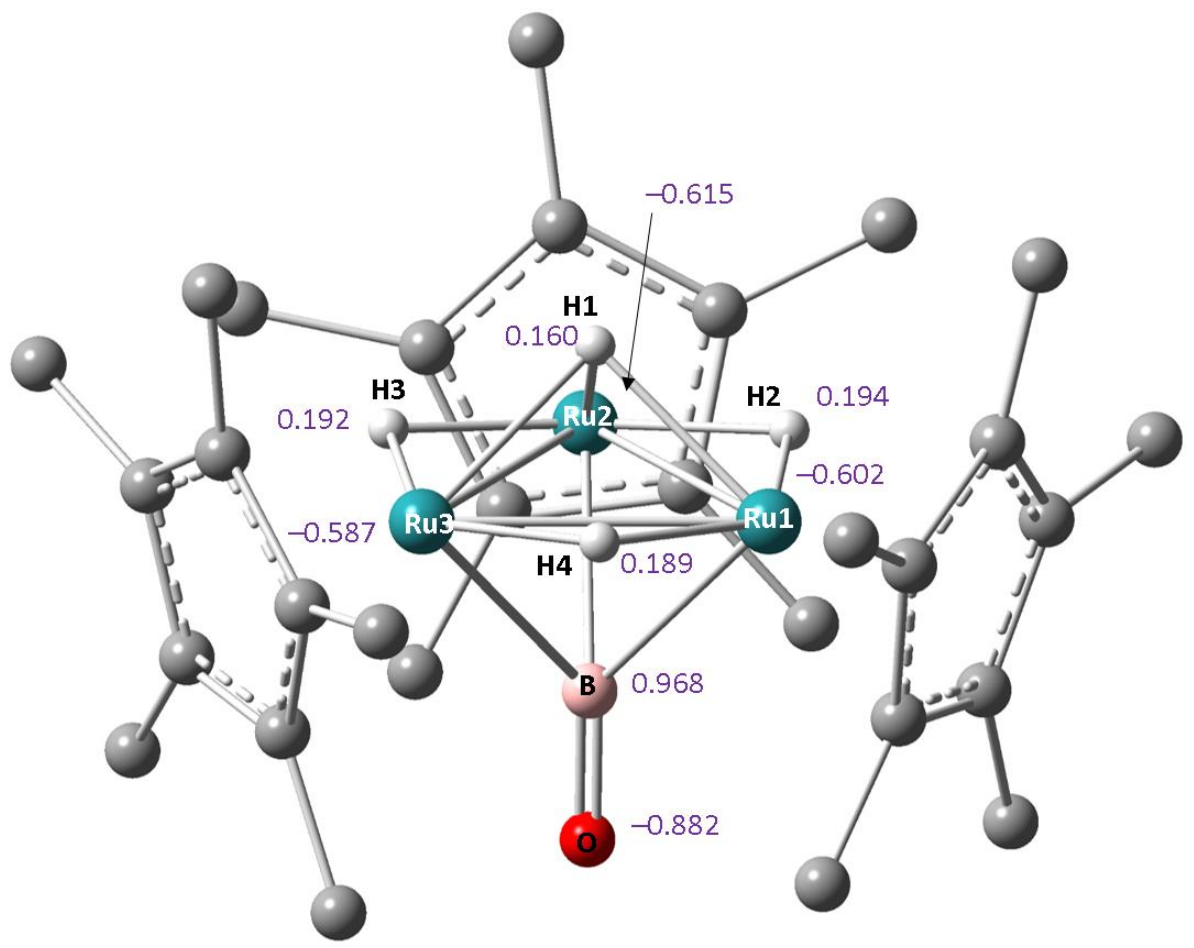

Figure S7. Optimized structure of $\left[\{\mathrm{Cp} * \mathrm{Ru}(\mu-\mathrm{H})\}_{3}\left(\mu_{3}-\mathrm{BO}\right)\left(\mu_{3}-\mathrm{H}\right)\right](3)$ by DFT calculations $(\omega \mathrm{B} 97 \mathrm{X}-$ $\mathrm{D} / \mathrm{SDD}(\mathrm{Ru}), 6-31 \mathrm{G}(\mathrm{d})(\mathrm{C}, \mathrm{H}, \mathrm{B}$, and $\mathrm{O}))$ and the values of NBO charge at the core atoms. The numbers written by the atoms indicate the values of NBO charge.

Table S8. Selected bond lengths $[\AA]$ and angles $\left[{ }^{\circ}\right]$ for 3. Corresponding X-ray data of $\mathbf{3}$ was shown for comparison.

\begin{tabular}{l|lll|ll}
\hline & 3' (Calcd.) & 3 (Measued) & & 3' (Calcd.) & 3 (Measued) \\
\hline \hline $\mathrm{Ru}(1)-\mathrm{Ru}(2)$ & 2.737 & $2.7113(4)$ & $\mathrm{Ru}(2)-\mathrm{H}(3)$ & 1.719 & $1.75(6)$ \\
$\mathrm{Ru}(1)-\mathrm{Ru}(3)$ & 2.733 & $2.7170(4)$ & $\mathrm{Ru}(3)-\mathrm{H}(3)$ & 1.734 & $1.71(6)$ \\
$\mathrm{Ru}(2)-\mathrm{Ru}(3)$ & 2.739 & $2.7205(5)$ & $\mathrm{Ru}(3)-\mathrm{H}(4)$ & 1.722 & $1.65(4)$ \\
$\mathrm{Ru}(1)-\mathrm{B}(1)$ & 2.311 & $2.302(5)$ & & & \\
$\mathrm{Ru}(2)-\mathrm{B}(1)$ & 2.322 & $2.320(6)$ & $\mathrm{Ru}(2)-\mathrm{Ru}(1)-\mathrm{Ru}(3)$ & 59.87 & $60.155(11)$ \\
$\mathrm{Ru}(3)-\mathrm{B}(1)$ & 2.317 & $2.305(5)$ & $\mathrm{Ru}(1)-\mathrm{Ru}(2)-\mathrm{Ru}(3)$ & 60.02 & $60.027(11)$ \\
$\mathrm{B}(1)-\mathrm{O}(1)$ & 1.242 & $1.231(6)$ & $\mathrm{Ru}(1)-\mathrm{Ru}(3)-\mathrm{Ru}(2)$ & 60.11 & $59.818(11)$ \\
$\mathrm{Ru}(1)-\mathrm{H}(1)$ & 1.976 & $1.97(6)$ & $\mathrm{Ru}(1)-\mathrm{B}(1)-\mathrm{Ru}(2)$ & 72.37 & $71.85(16)$ \\
$\mathrm{Ru}(2)-\mathrm{H}(1)$ & 1.962 & $1.94(6)$ & $\mathrm{Ru}(1)-\mathrm{B}(1)-\mathrm{Ru}(3)$ & 72.41 & $72.29(16)$ \\
$\mathrm{Ru}(3)-\mathrm{H}(1)$ & 1.995 & $1.91(7)$ & $\mathrm{Ru}(1)-\mathrm{B}(1)-\mathrm{O}(1)$ & 136.71 & $137.5(4)$ \\
$\mathrm{Ru}(1)-\mathrm{H}(2)$ & 1.722 & $1.74(5)$ & $\mathrm{Ru}(2)-\mathrm{B}(1)-\mathrm{Ru}(3)$ & 72.38 & $72.07(16)$ \\
$\mathrm{Ru}(1)-\mathrm{H}(4)$ & 1.731 & $1.67(4)$ & $\mathrm{Ru}(2)-\mathrm{B}(1)-\mathrm{O}(1)$ & 137.05 & $136.5(4)$ \\
$\mathrm{Ru}(2)-\mathrm{H}(2)$ & 1.729 & $1.65(5)$ & $\mathrm{Ru}(3)-\mathrm{B}(1)-\mathrm{O}(1)$ & 137.26 & $137.6(4)$ \\
\hline
\end{tabular}


Top view

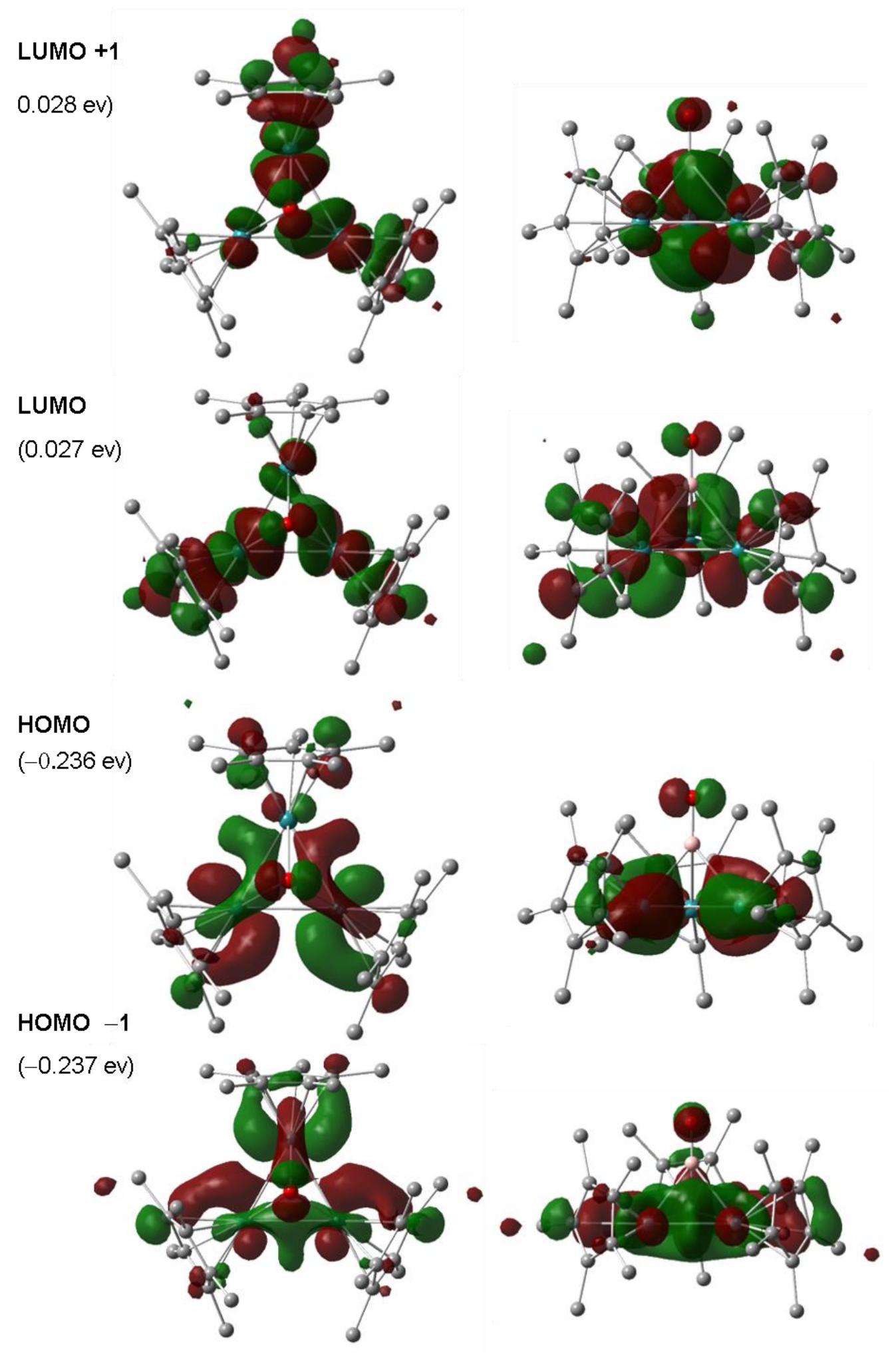

Figure S8. The shapes of the LUMO+1, LUMO, HOMO, and HOMO-1 of 3 with an isovalue of 0.03 . 


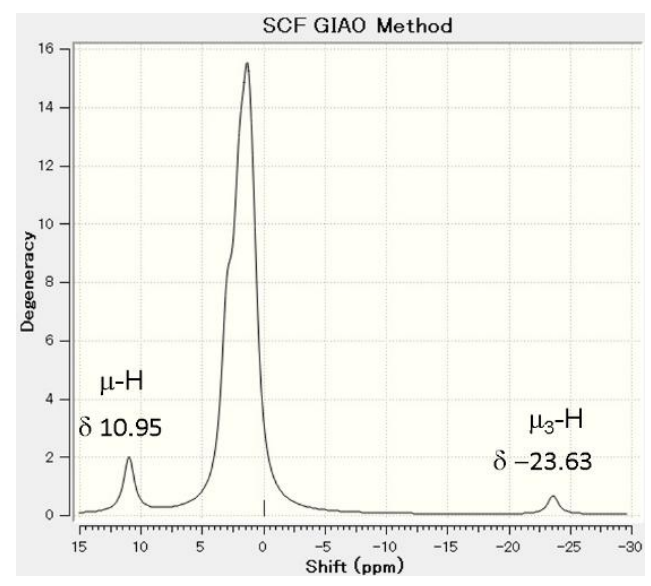

Figure S9. Calculated ${ }^{1} \mathrm{H}$ NMR spectrum for 3 by Gauge-Independent Atomic Orbital method at the $\omega \mathrm{B} 97 \mathrm{X}-\mathrm{D} / \mathrm{Ru}$ : SDD $, \mathrm{C}, \mathrm{H}, \mathrm{B}, \mathrm{O}: 6-31 \mathrm{G}(\mathrm{d})$ level of theory. The reference shielding was set at $0 \mathrm{ppm}$ (SiMe4, B3LYP/6-311+G(2d,p) GIAO).

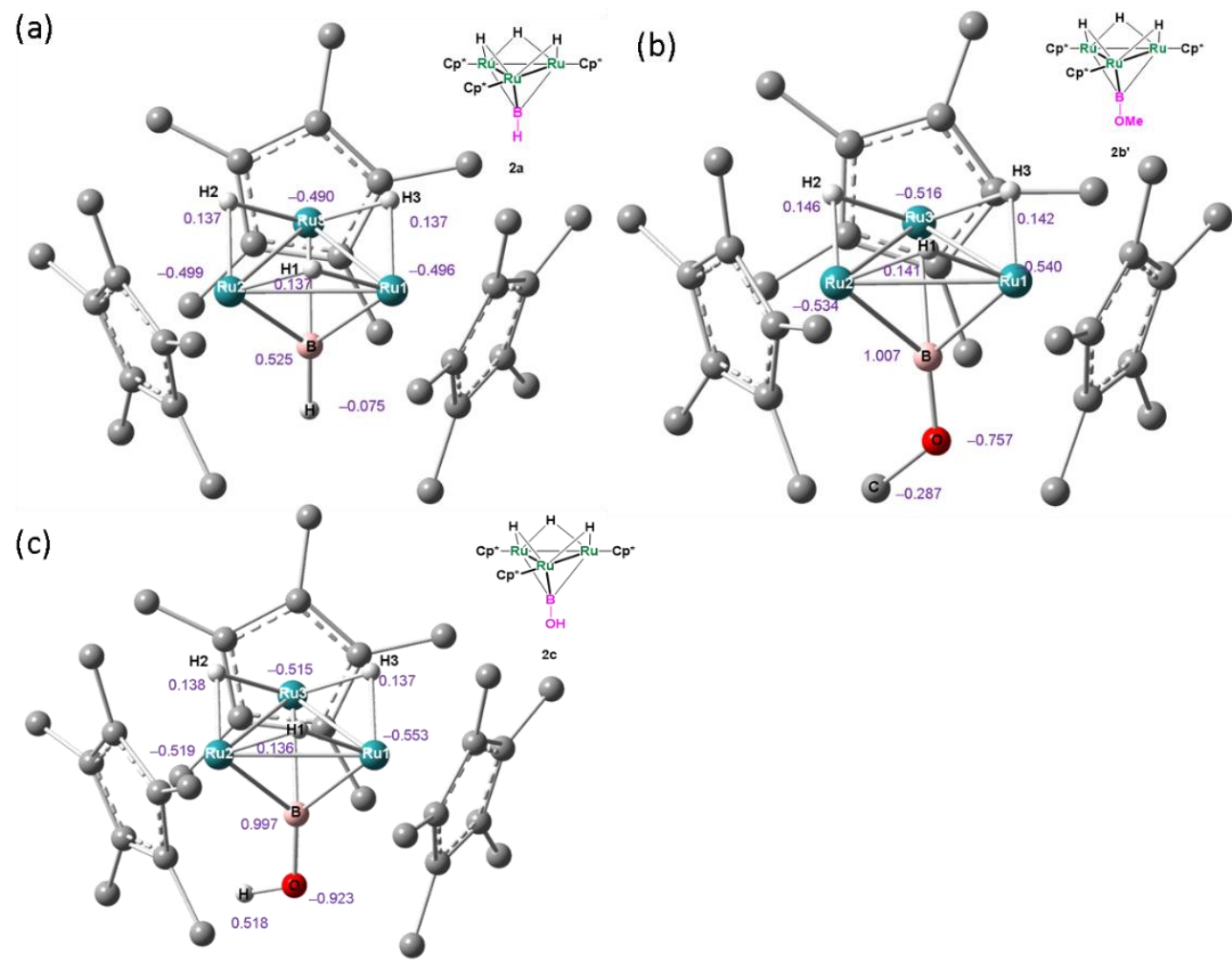

Figure S10. Optimized structure of (a) $\left[\{\mathrm{Cp} * \mathrm{Ru}(\mu-\mathrm{H})\}_{3}\left(\mu_{3}-\mathrm{BH}\right)\right]$ (2a), (b) $\left[\{\mathrm{Cp} * \mathrm{Ru}(\mu-\mathrm{H})\}_{3}\left(\mu_{3}-\right.\right.$ BOMe)] (2b'), (c) $\left[\{\mathrm{Cp} * \mathrm{Ru}(\mu-\mathrm{H})\}_{3}\left(\mu_{3}-\mathrm{BOH}\right)\right](\mathbf{2})$ by DFT calculations $(\omega \mathrm{B} 97 \mathrm{X}-\mathrm{D} / \mathrm{SDD}(\mathrm{Ru}), 6-$ $31 \mathrm{G}(\mathrm{d})(\mathrm{C}, \mathrm{H}, \mathrm{B}$, and $\mathrm{O}))$ and the values of NBO charge at the core atoms. The numbers written by the atoms indicate the values of NBO charge. 
Table S9. Selected bond lengths $(\AA)$ and angles (Deg) for the optimized structures of $[\{\mathrm{Cp} * \mathrm{Ru}(\mu-$ $\left.\mathrm{H})\}_{3}\left(\mu_{3}-\mathrm{BH}\right)\right] \quad(\mathbf{2 a}), \quad\left[\{\mathrm{Cp} * \mathrm{Ru}(\mu-\mathrm{H})\}_{3}\left(\mu_{3}-\mathrm{BOMe}\right)\right] \quad\left(\mathbf{2} \mathbf{b}^{\prime}\right)$, and $\left[\{\mathrm{Cp} * \mathrm{Ru}(\mu-\mathrm{H})\}_{3}\left(\mu_{3}-\mathrm{BOH}\right)\right] \quad(\mathbf{2 c})$. Corresponding X-ray data of $\left[\{\mathrm{Cp} * \mathrm{Ru}(\mu-\mathrm{H})\}_{3}\left(\mu_{3}-\mathrm{BH}\right)\right](\mathbf{2 a})$ and $\left[\{\mathrm{Cp} * \mathrm{Ru}(\mu-\mathrm{H})\}_{3}\left(\mu_{3}-\mathrm{BOEt}\right)\right](\mathbf{2 b})$ was also shown for comparison.

\begin{tabular}{|c|c|c|c|c|c|}
\hline & 2a (calcd.) & 2b' (calcd.) & 2c (calcd.) & 2a (measured) & $\mathbf{2 b}$ (measured) \\
\hline $\mathrm{Ru} 1-\mathrm{Ru} 2$ & 2.674 & 2.686 & 2.687 & $2.6529(8)$ & $2.6769(6)$ \\
\hline Ru1-Ru3 & 2.677 & 2.692 & 2.686 & $2.6538(8)$ & $2.6769(6)$ \\
\hline Ru2-Ru3 & 2.675 & 2.688 & 2.695 & $2.6539(8)$ & $2.6781(5)$ \\
\hline Ru1-B & 2.123 & 2.125 & 2.116 & $2.120(9)$ & $2.177(6)$ \\
\hline Ru1-H1 & 1.754 & 1.751 & 1.750 & $1.68(9)$ & $1.59(6)$ \\
\hline Ru1-H3 & 1.753 & 1.748 & 1.749 & $1.77(12)$ & $1.76(5)$ \\
\hline $\mathrm{Ru} 2-\mathrm{B}$ & 2.126 & 2.205 & 2.180 & $2.108(10)$ & $2.140(7)$ \\
\hline $\mathrm{Ru} 2-\mathrm{H} 1$ & 1.755 & 1.746 & 1.757 & $1.68(9)$ & $1.66(6)$ \\
\hline $\mathrm{Ru} 2-\mathrm{H} 2$ & 1.746 & 1.732 & 1.752 & $1.75(11)$ & $1.70(6)$ \\
\hline Ru3-B & 2.125 & 2.212 & 2.188 & $2.120(8)$ & $2.145(6)$ \\
\hline Ru3-H2 & 1.761 & 1.751 & 1.749 & $1.74(11)$ & $1.72(6)$ \\
\hline Ru3-H3 & 1.754 & 1.751 & 1.757 & $1.77(12)$ & $1.71(5)$ \\
\hline $\mathrm{B}-\mathrm{O}$ & & 1.353 & 1.367 & & $1.374(13)$ \\
\hline B-H & 1.208 & & & $1.24(15)$ & \\
\hline $\mathrm{O}-\mathrm{C}$ & & 1.405 & & & $1.43(3)$ \\
\hline $\mathrm{O}-\mathrm{H}$ & & & 0.965 & & \\
\hline Ru2-Ru1-Ru3 & 59.99 & 59.96 & 60.20 & $60.01(2)$ & $60.030(14)$ \\
\hline Ru1-Ru2-Ru3 & 60.05 & 60.14 & 59.88 & $60.01(2)$ & $59.984(15)$ \\
\hline Ru1-Ru3-Ru2 & 59.95 & 59.90 & 59.92 & $59.98(2)$ & $59.986(15)$ \\
\hline $\mathrm{Ru} 2-\mathrm{Ru} 1-\mathrm{B}$ & 51.06 & 53.00 & 52.36 & $50.9(3)$ & $51.06(18)$ \\
\hline Ru3-Ru1-B & 50.98 & 53.09 & 52.61 & $51.3(2)$ & $51.20(17)$ \\
\hline Ru1-Ru2-B & 50.95 & 50.33 & 50.21 & $51.3(3)$ & $52.31(17)$ \\
\hline Ru3-Ru2-B & 50.99 & 52.64 & 52.05 & $51.3(2)$ & 51.41(17) \\
\hline Ru1-Ru3-B & 50.91 & 50.18 & 50.18 & $51.2(2)$ & $52.27(17)$ \\
\hline $\mathrm{Ru} 2-\mathrm{Ru} 3-\mathrm{B}$ & 51.03 & 52.40 & 51.77 & $50.9(3)$ & $51.24(18)$ \\
\hline Ru1-B-Ru2 & 77.98 & 76.67 & 77.42 & $77.7(3)$ & $76.6(2)$ \\
\hline Ru1-B-Ru3 & 78.10 & 76.73 & 77.21 & $77.5(3)$ & $76.5(2)$ \\
\hline Ru1-B-O & & 129.74 & 135.56 & & $139.3(7)$ \\
\hline Ru1-B-H & 133.40 & & & $129(8)$ & \\
\hline $\mathrm{Ru} 2-\mathrm{B}-\mathrm{Ru} 3$ & 77.98 & 74.97 & 76.18 & $77.8(3)$ & $77.4(2)$ \\
\hline Ru2-B-O & & 137.39 & 133.25 & & 121.3(9) \\
\hline Ru2-B-H & 133.39 & & & $140(8)$ & \\
\hline Ru3-B-O & & 136.57 & 133.37 & & $139.6(9)$ \\
\hline Ru3-B-H & 133.35 & & & $131(7)$ & \\
\hline
\end{tabular}




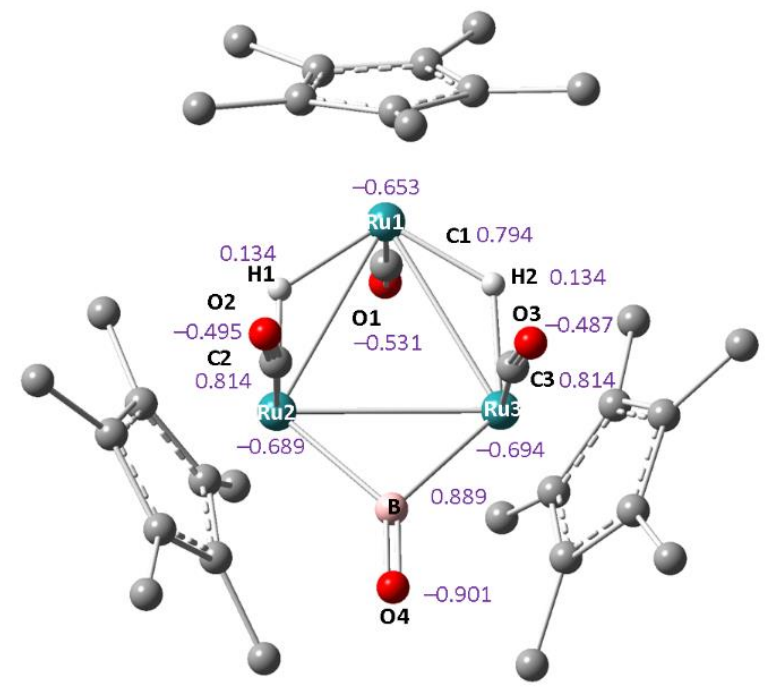

Figure S11. Optimized structure of $\left[\{\mathrm{Cp} * \mathrm{Ru}(\mathrm{CO})\}_{3}(\mu-\mathrm{BO})(\mu-\mathrm{H})_{2}\right](7)$ by DFT calculations $(\omega \mathrm{B} 97 \mathrm{X}-\mathrm{D} / \mathrm{SDD}(\mathrm{Ru}), 6-31 \mathrm{G}(\mathrm{d})(\mathrm{C}, \mathrm{H}, \mathrm{B}$, and $\mathrm{O}))$ and the values of NBO charge at the core atoms. The numbers written by the atoms indicate the values of NBO charge.

Table S10. Selected bond lengths $(\AA)$ and angles (Deg) for the optimized structures of 7 . Corresponding X-ray data of $\left[\{\mathrm{Cp} * \mathrm{Ru}(\mathrm{CO})\}_{3}(\mu-\mathrm{BO})(\mu-\mathrm{H})_{2}\right](7)$ was also shown for comparison.

\begin{tabular}{c|ccc|c|c}
\hline \multicolumn{2}{c}{ Lengths } & calcd. & measured & Angles & \multicolumn{2}{c}{ calcd. } & Measured \\
\hline \hline Ru1-Ru2 & 3.035 & $3.0140(4)$ & Ru2-Ru1-Ru3 & 58.54 & $58.741(9)$ \\
Ru1-Ru3 & 3.044 & $3.0167(4)$ & Ru1-Ru2-Ru3 & 60.88 & $60.675(9)$ \\
Ru2-Ru3 & 2.973 & $2.9578(4)$ & Ru1-Ru3-Ru2 & 60.57 & $60.585(9)$ \\
Ru1-C1 & 1.833 & $1.845(4)$ & Ru2-Ru1-C1 & 91.59 & $91.28(12)$ \\
Ru1-H1 & 1.692 & $1.62(6)$ & Ru3-Ru1-C1 & 90.11 & $87.38(11)$ \\
Ru1-H2 & 1.700 & $1.62(6)$ & Ru1-Ru2-B & 106.16 & $106.61(12)$ \\
Ru2-B & 2.180 & $2.194(4)$ & Ru1-Ru2-C2 & 89.37 & $86.78(12)$ \\
Ru2-C2 & 1.840 & $1.850(4)$ & Ru3-Ru2-B & 46.68 & $47.09(12)$ \\
Ru2-H1 & 1.731 & $1.63(6)$ & Ru3-Ru2-C2 & 91.68 & $87.97(12)$ \\
Ru3-B & 2.167 & $2.174(5)$ & Ru1-Ru3-B & 106.20 & $107.08(11)$ \\
Ru3-C3 & 1.844 & $1.853(4)$ & Ru2-Ru3-B & 47.04 & $47.66(11)$ \\
Ru3-H2 & 1.725 & $1.70(6)$ & Ru1-Ru3-C3 & 92.79 & $95.42(12)$ \\
B-O4 & 1.244 & $1.236(5)$ & Ru2-Ru3-C3 & 93.73 & $93.14(12)$ \\
C1-O1 & 1.167 & $1.152(5)$ & Ru2-B-Ru3 & 86.27 & $85.26(16)$ \\
C2-O2 & 1.160 & $1.143(5)$ & Ru2-B-O4 & 136.56 & $137.9(3)$ \\
C3-O3 & 1.158 & $1.143(5)$ & Ru3-B-O4 & 137.13 & $136.9(3)$ \\
\hline
\end{tabular}

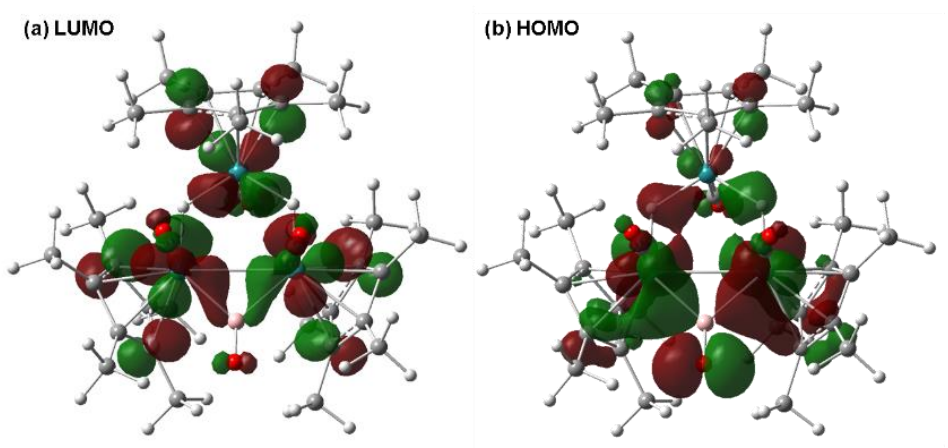

Figure S12. The shapes of the LUMO $(-0.001 \mathrm{eV})$ and HOMO $(-0.249 \mathrm{eV})$ of 7 with an isovalue of 0.03 . 
(a)

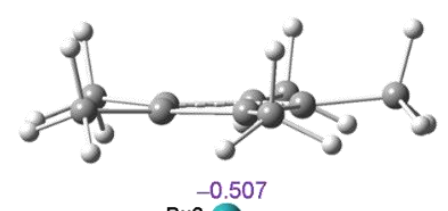

(b)

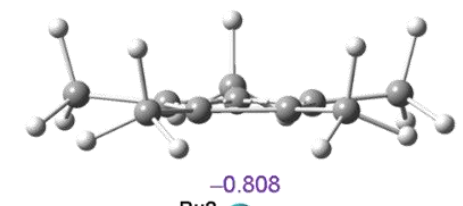

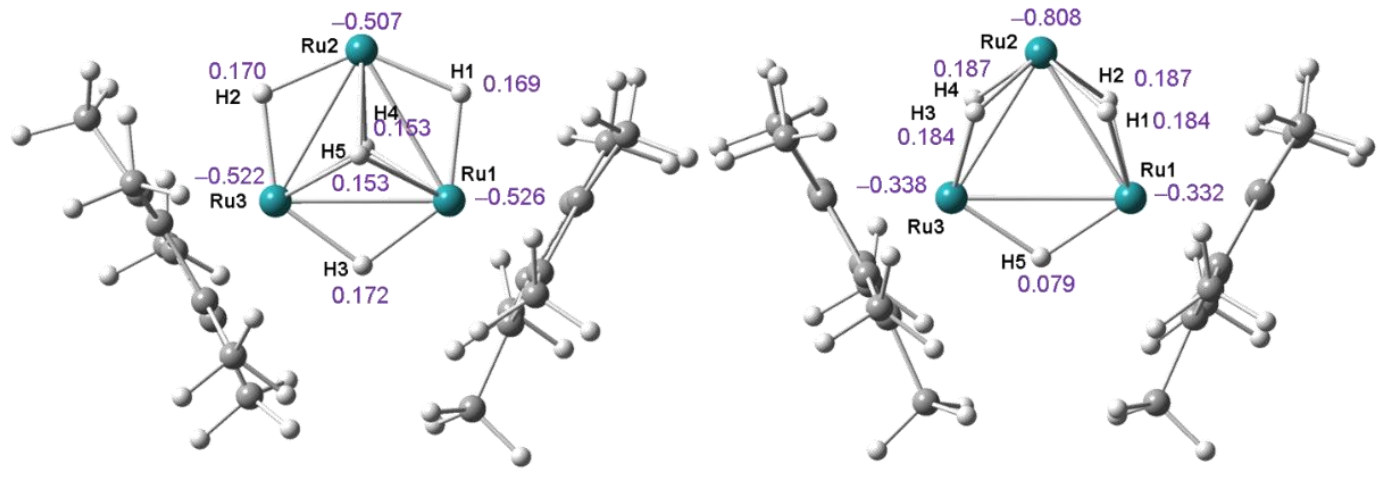

Figure S13. Optimized structure of $\mathbf{4}$ and its isomer $\mathbf{D}$ by DFT calculations ( $\omega$ B97X-D /SDD (Ru), 6$31 \mathrm{G}(\mathrm{d})(\mathrm{C}$ and $\mathrm{O})$ ) and the values of NBO charge at the core atoms. The numbers written by the atoms indicate the values of NBO charge.

Table S11. Selected bond lengths $(\AA)$ and angles (Deg) for the optimized structures of 4 and D. Corresponding X-ray data of $\left[\{\mathrm{Cp} * \mathrm{Ru}(\mu-\mathrm{H})\}_{3}\left(\mu_{3}-\mathrm{H}\right)_{2}\right](4)$ was also shown for comparison.

\begin{tabular}{c|ccc}
\hline & 4 (calcd.) & 4 (Measured) & D \\
\hline \hline Ru1-Ru2 & 2.775 & $2.7534(8)$ & 2.733 \\
Ru1-Ru3 & 2.774 & $2.7503(8)$ & 2.864 \\
Ru2-Ru3 & 2.771 & $2.7453(6)$ & 2.733 \\
Ru- $\mu-H$ (av). & 1.73 & 1.66 & --- \\
Ru- $\mu_{3}-\mathrm{H}$ (av) & 1.96 & 1.94 & --- \\
& & & \\
Ru2-Ru1-Ru3 & 59.92 & $59.844(18)$ & 58.40 \\
Ru1-Ru2-Ru3 & 60.02 & $60.02(2)$ & 63.20 \\
Ru1-Ru3-Ru2 & 60.05 & $60.135(18)$ & 58.41 \\
\hline
\end{tabular}

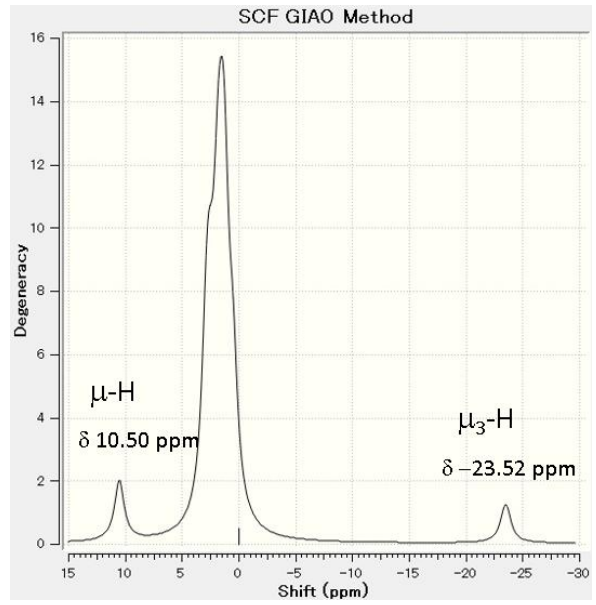

Figure S14. Calculated ${ }^{1} \mathrm{H}$ NMR spectrum for 4 by Gauge-Independent Atomic Orbital method at the $\omega$ B97X-D/Ru: SDD, C, H, B, O: 6-31G(d) level of theory. The reference shielding was set at 0 ppm (SiMe4, B3LYP/6-311+G(2d,p) GIAO). 


\section{NMR and IR spectra of compounds}

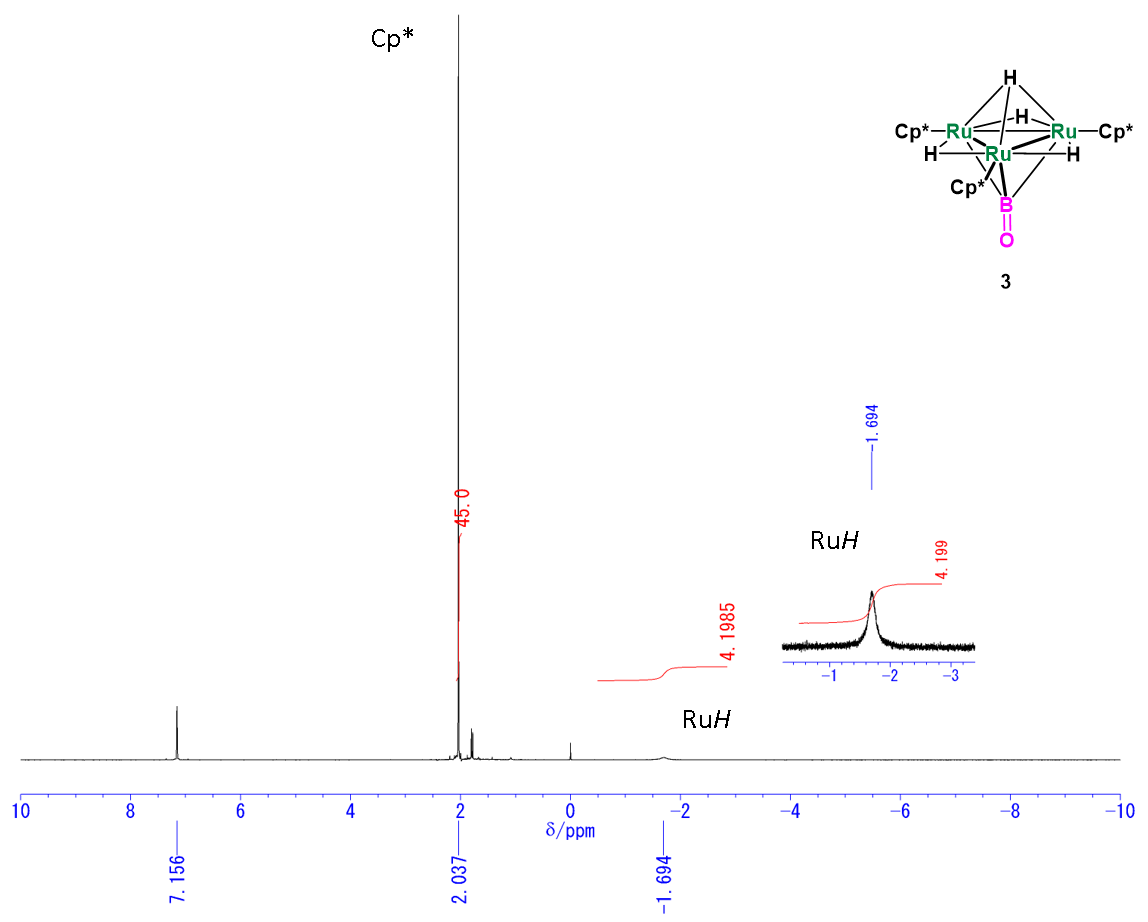

Figure S15. ${ }^{1} \mathrm{H}$ NMR spectrum of $3\left(400 \mathrm{MHz}, \mathrm{C}_{6} \mathrm{D}_{6}, 25^{\circ} \mathrm{C}\right)$.

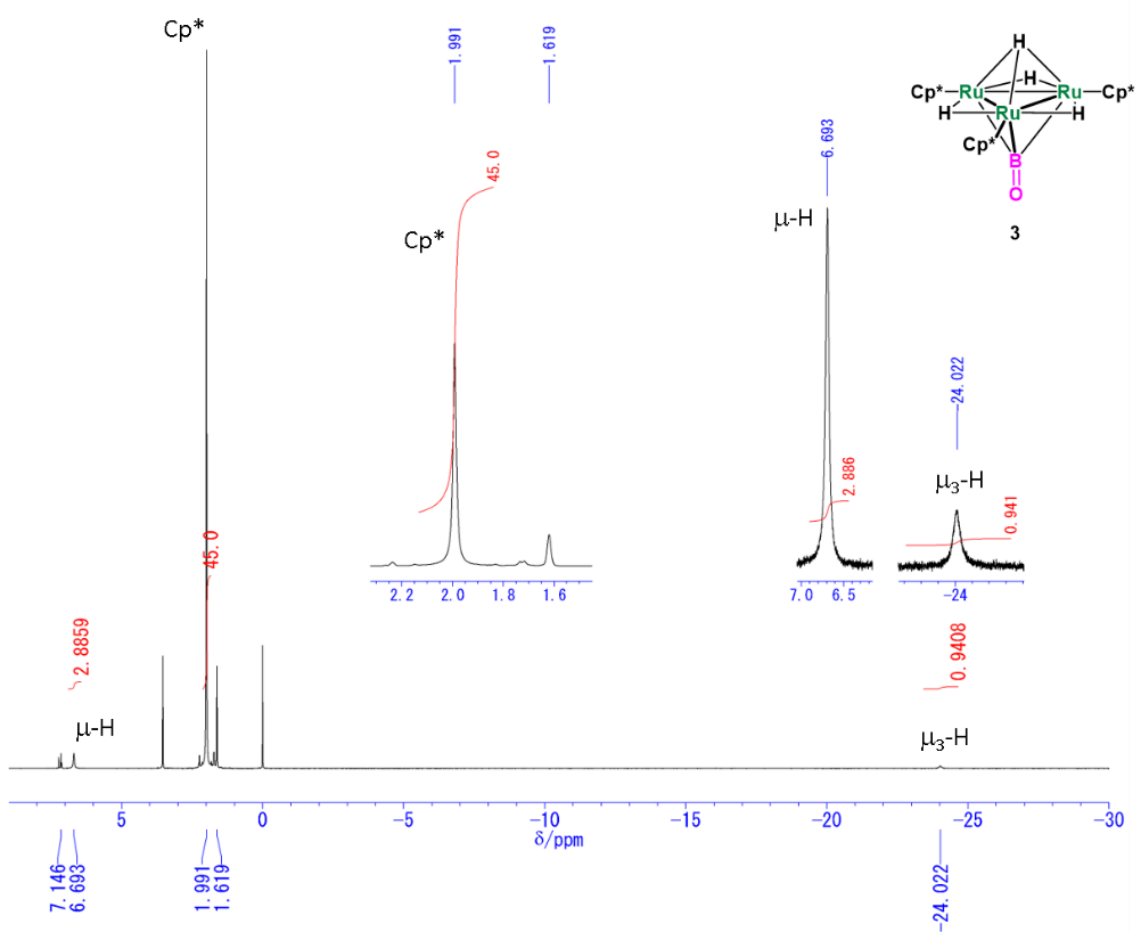

Figure S16. ${ }^{1} \mathrm{H}$ NMR spectrum of $\mathbf{3}\left(400 \mathrm{MHz}\right.$, THF- $d_{8}:$ toluene- $\left.d_{8}=4: 1,-110{ }^{\circ} \mathrm{C}\right)$. 


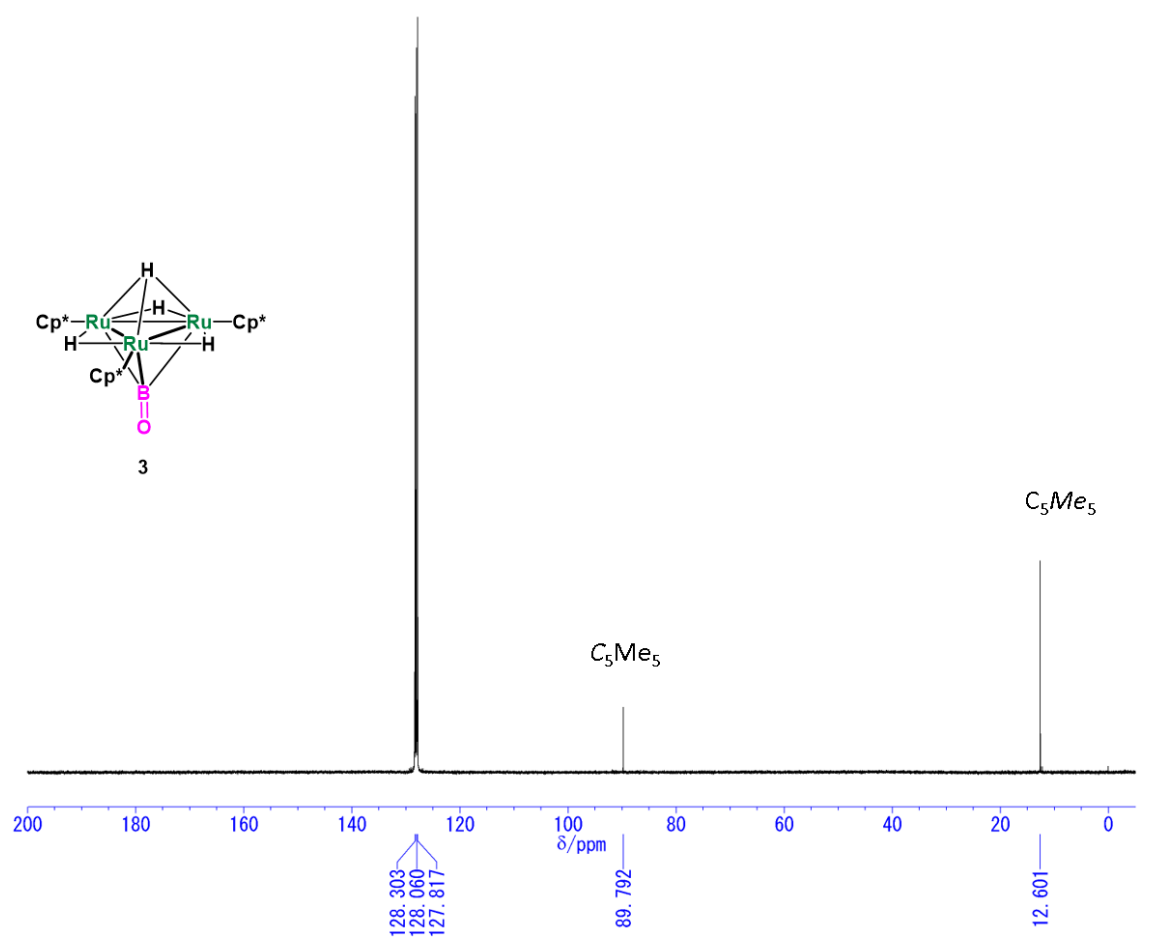

Figure S17. ${ }^{13} \mathrm{C}\left\{{ }^{1} \mathrm{H}\right\}$ NMR spectrum of $3\left(100 \mathrm{MHz}, \mathrm{C}_{6} \mathrm{D}_{6}, 25^{\circ} \mathrm{C}\right)$.

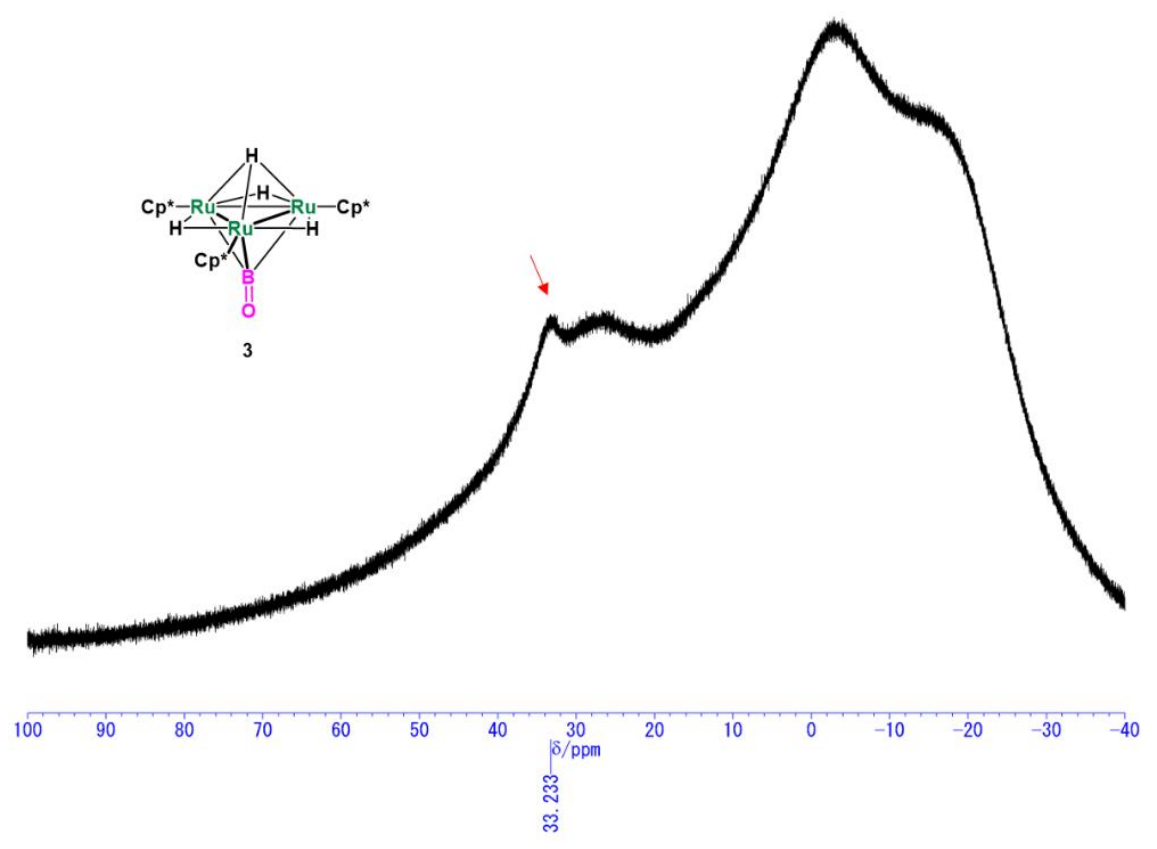

Figure S18. ${ }^{11} \mathrm{~B}\left\{{ }^{1} \mathrm{H}\right\}$ NMR spectrum of $\mathbf{3}\left(129 \mathrm{MHz}, \mathrm{C}_{6} \mathrm{D}_{6}, 2{ }^{\circ} \mathrm{C}\right)$. 


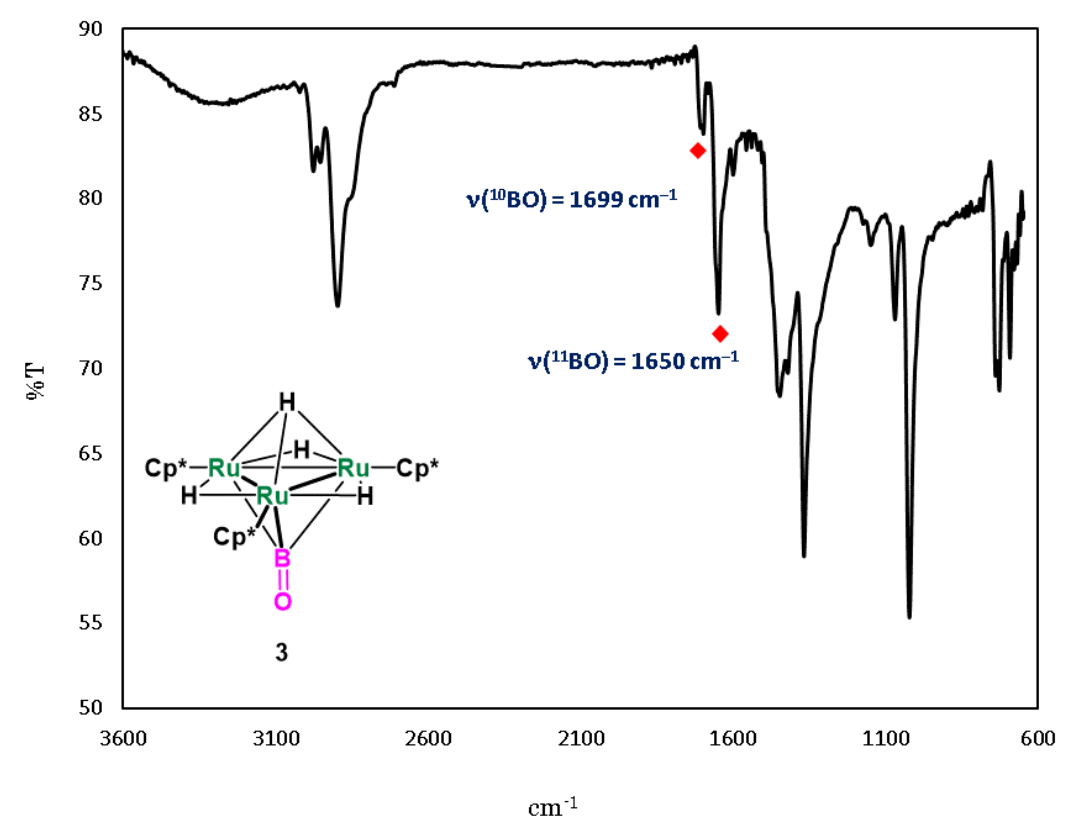

Figure S19. IR spectrum of $\mathbf{3}\left(\mathrm{ATR}, \mathrm{cm}^{-1}\right)$.
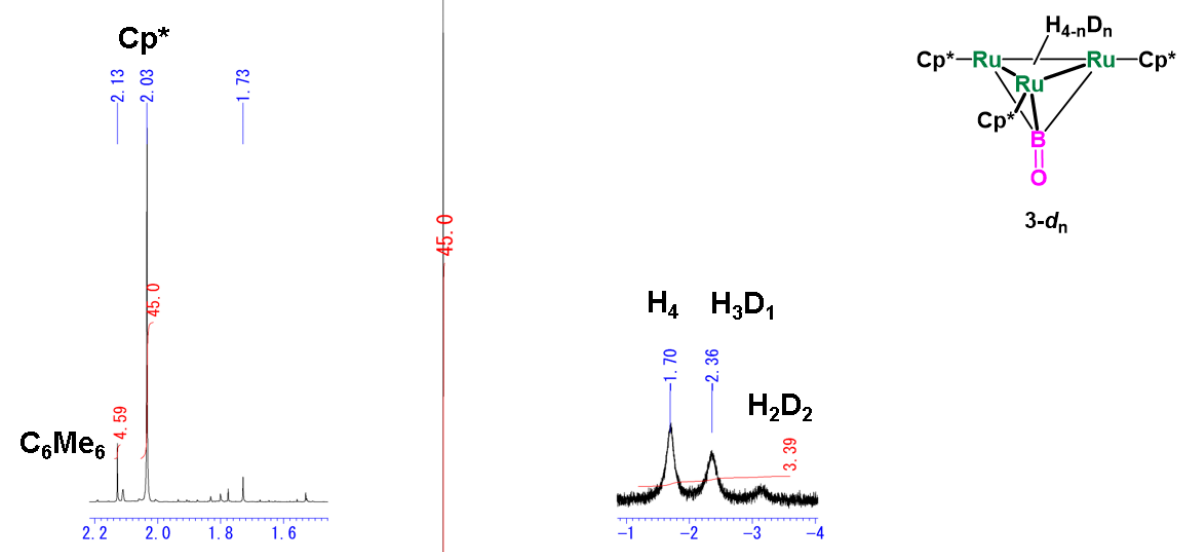

$\mathrm{H}_{4} \quad \mathrm{H}_{3} \mathrm{D}_{1}$
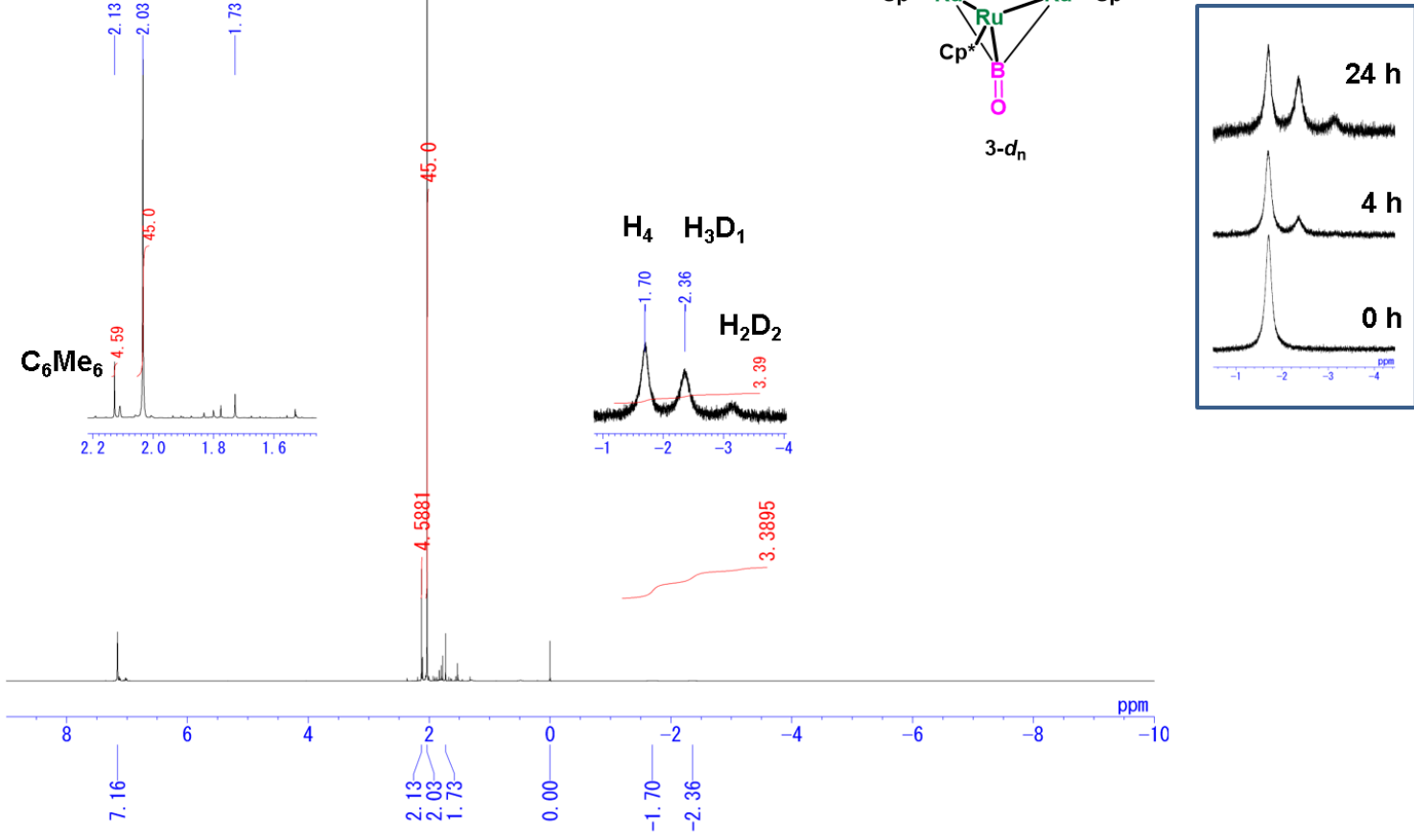

Figure S20. ${ }^{1} \mathrm{H}$ NMR spectrum of partially deuterated 3 obtained by heating 3 in $\mathrm{C}_{6} \mathrm{D}_{6}$ at $70{ }^{\circ} \mathrm{C}$ for 24 $\mathrm{h}\left(400 \mathrm{MHz}, \mathrm{C}_{6} \mathrm{D}_{6}, 25^{\circ} \mathrm{C}\right)$. The spectra in the square shows the time-course of the change of the hydrido signal. 


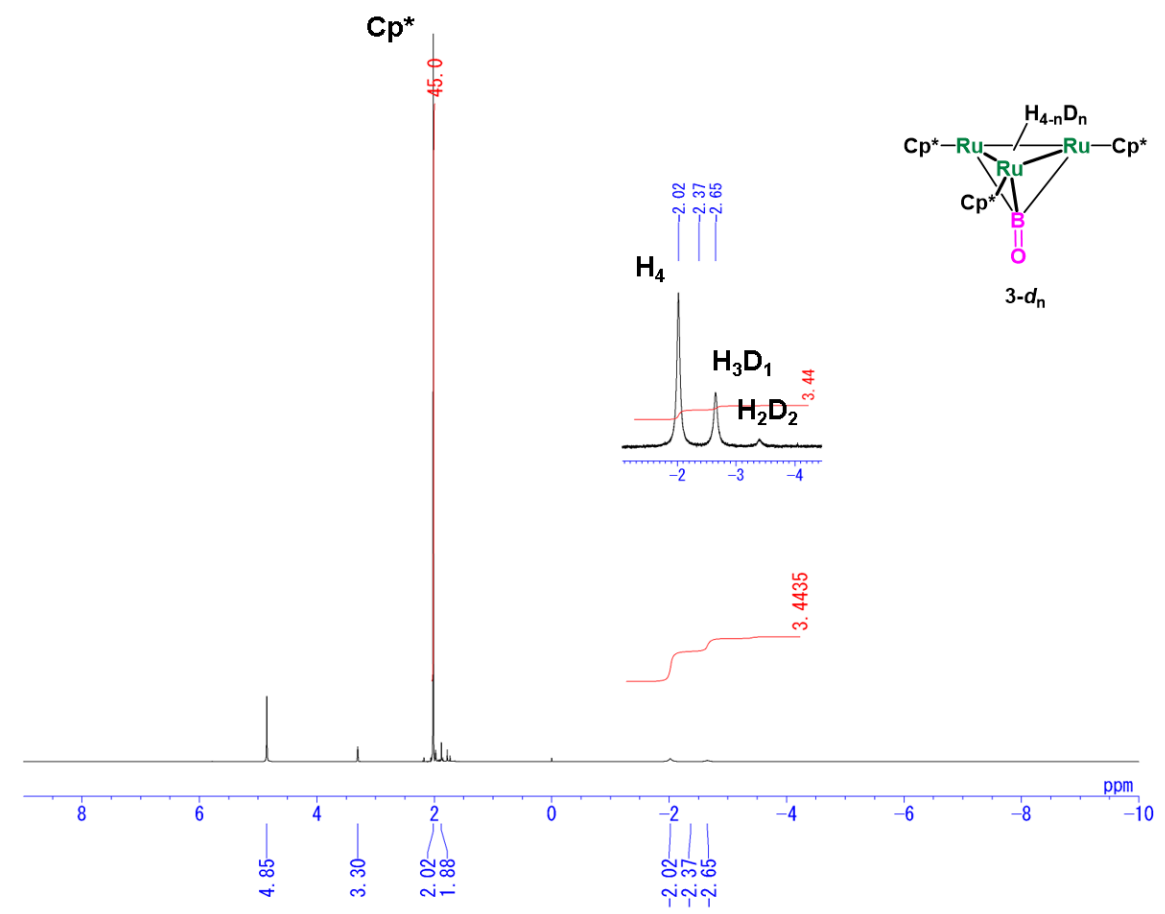

Figure S21. ${ }^{1} \mathrm{H}$ NMR spectrum of deuterated $\mathbf{3}$ obtained by the reaction of $\mathbf{3}$ with $\mathrm{CD}_{3} \mathrm{OD}$ at $25{ }^{\circ} \mathrm{C}$ for 6 days $\left(400 \mathrm{MHz}, \mathrm{CD}_{3} \mathrm{COD}, 25^{\circ} \mathrm{C}\right)$.

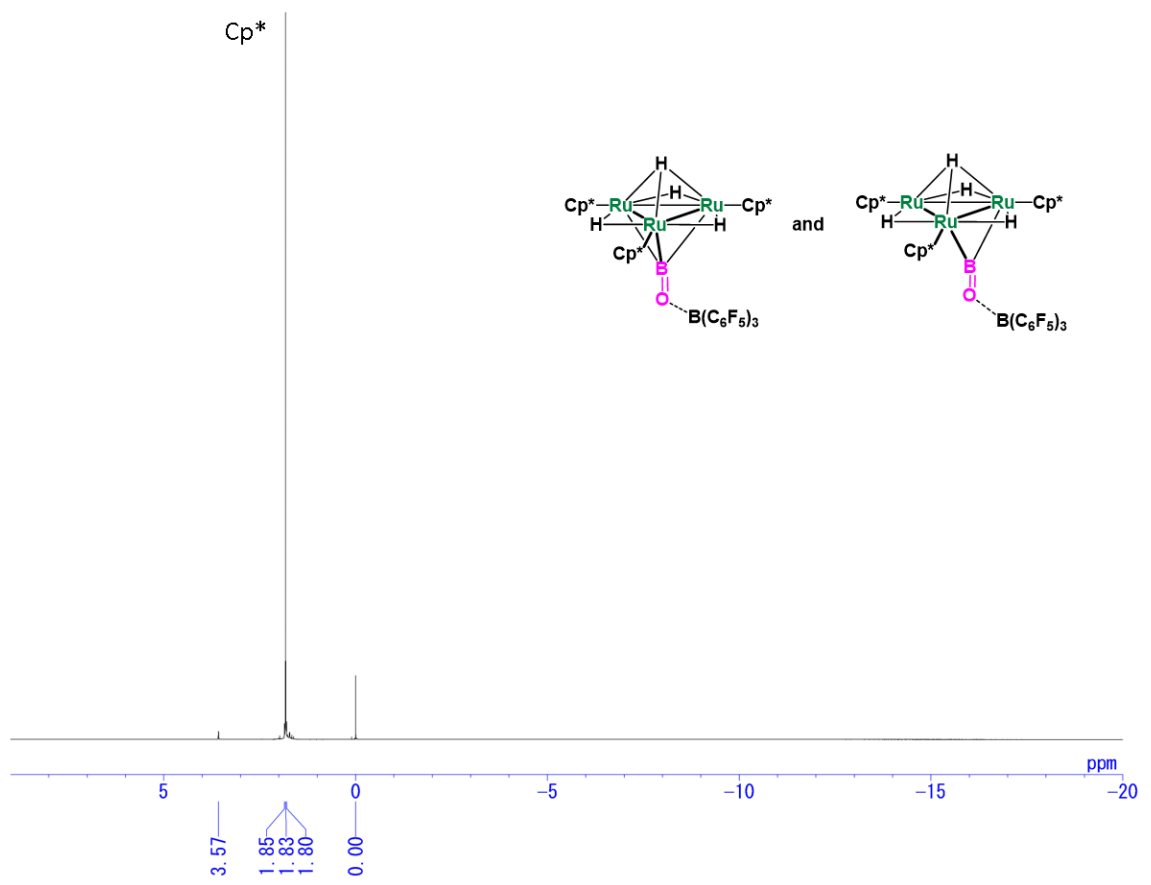

Figure S22. ${ }^{1} \mathrm{H}$ NMR spectrum of $5\left(400 \mathrm{MHz}, \mathrm{THF}-d_{8}, 25^{\circ} \mathrm{C}\right)$. 


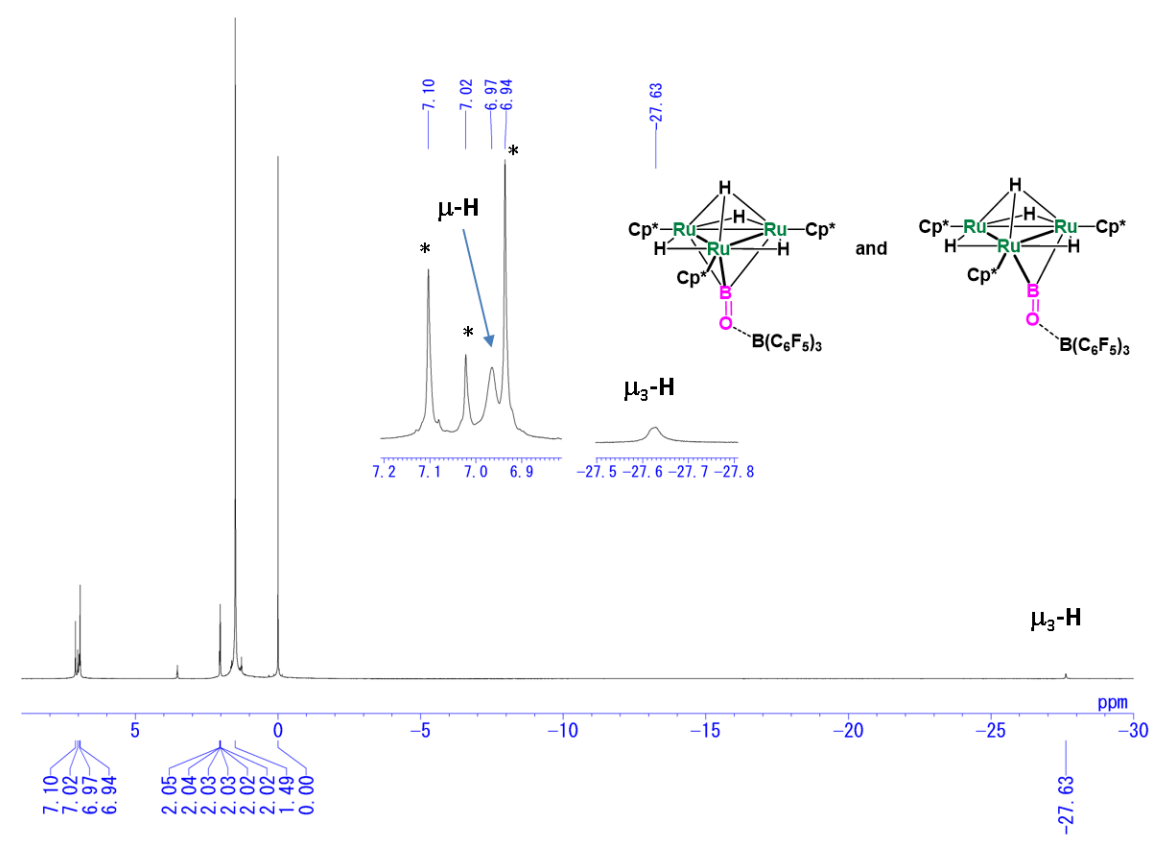

Figure S23. ${ }^{1} \mathrm{H}$ NMR spectrum of $5\left(400 \mathrm{MHz}\right.$, toluene- $\left.d_{8},-80{ }^{\circ} \mathrm{C}\right)$. The signals with asterisk were derived from toluene- $d_{8}$.

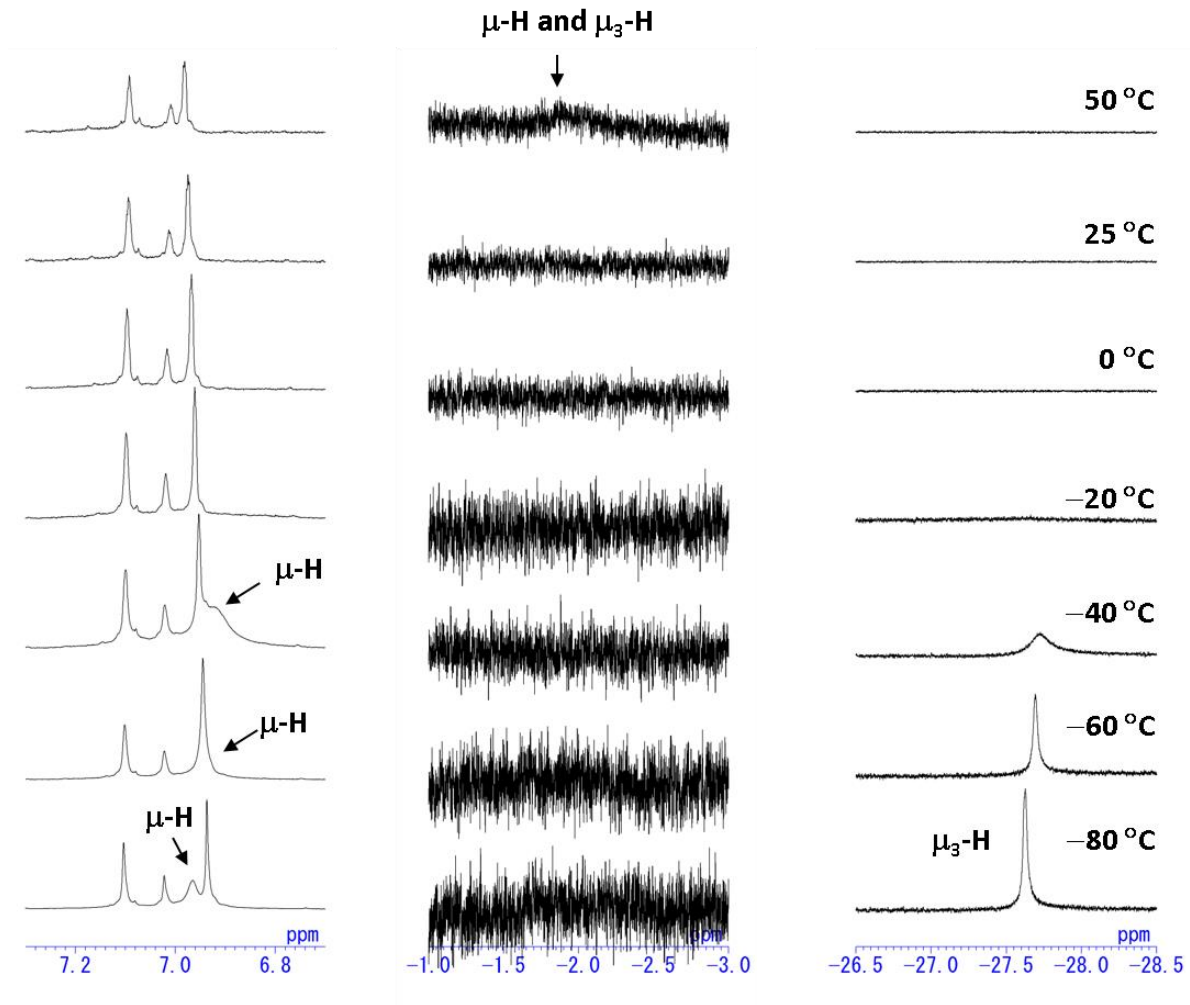

Figure S24. Variable temperature ${ }^{1} \mathrm{H}$ NMR spectra of $5\left(400 \mathrm{MHz}\right.$, toluene- $\left.d_{8}\right)$. 


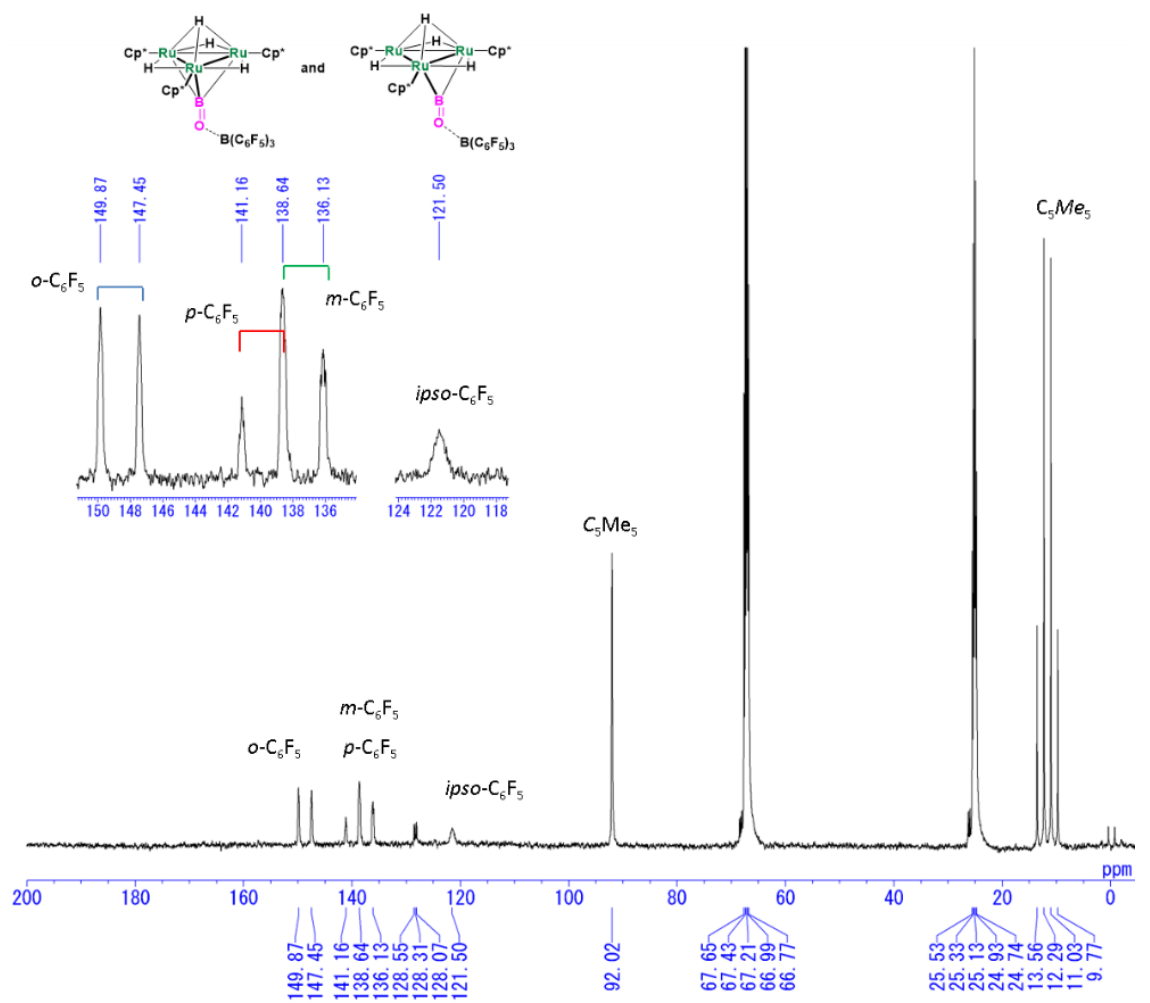

Figure S25. ${ }^{13} \mathrm{C}$ NMR spectrum of $5\left(100 \mathrm{MHz}, \mathrm{THF}-d_{8}, 25^{\circ} \mathrm{C}\right)$.

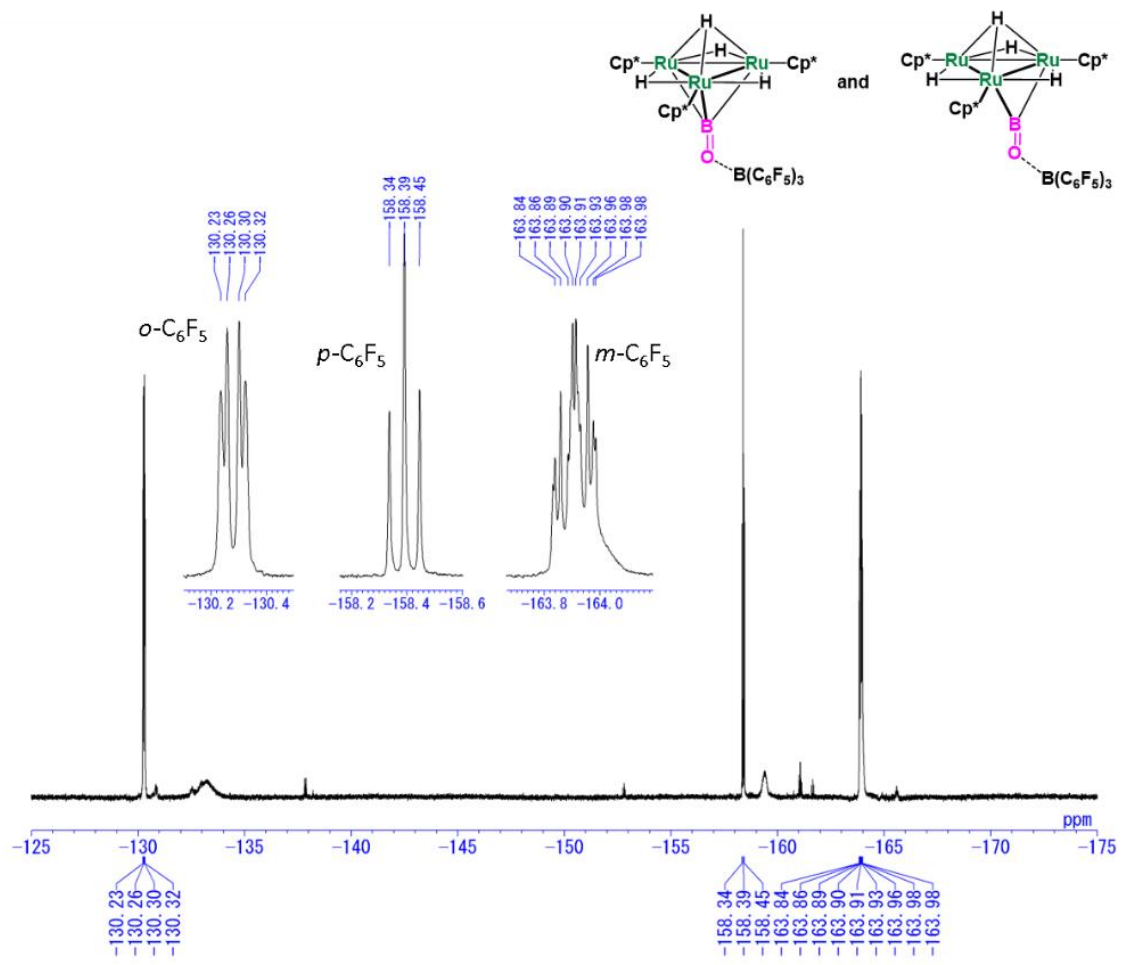

Figure S26. ${ }^{19} \mathrm{~F}\left\{{ }^{1} \mathrm{H}\right\}$ NMR spectrum of $5\left(375 \mathrm{MHz}, \mathrm{C}_{6} \mathrm{D}_{6}, 25^{\circ} \mathrm{C}\right)$. 


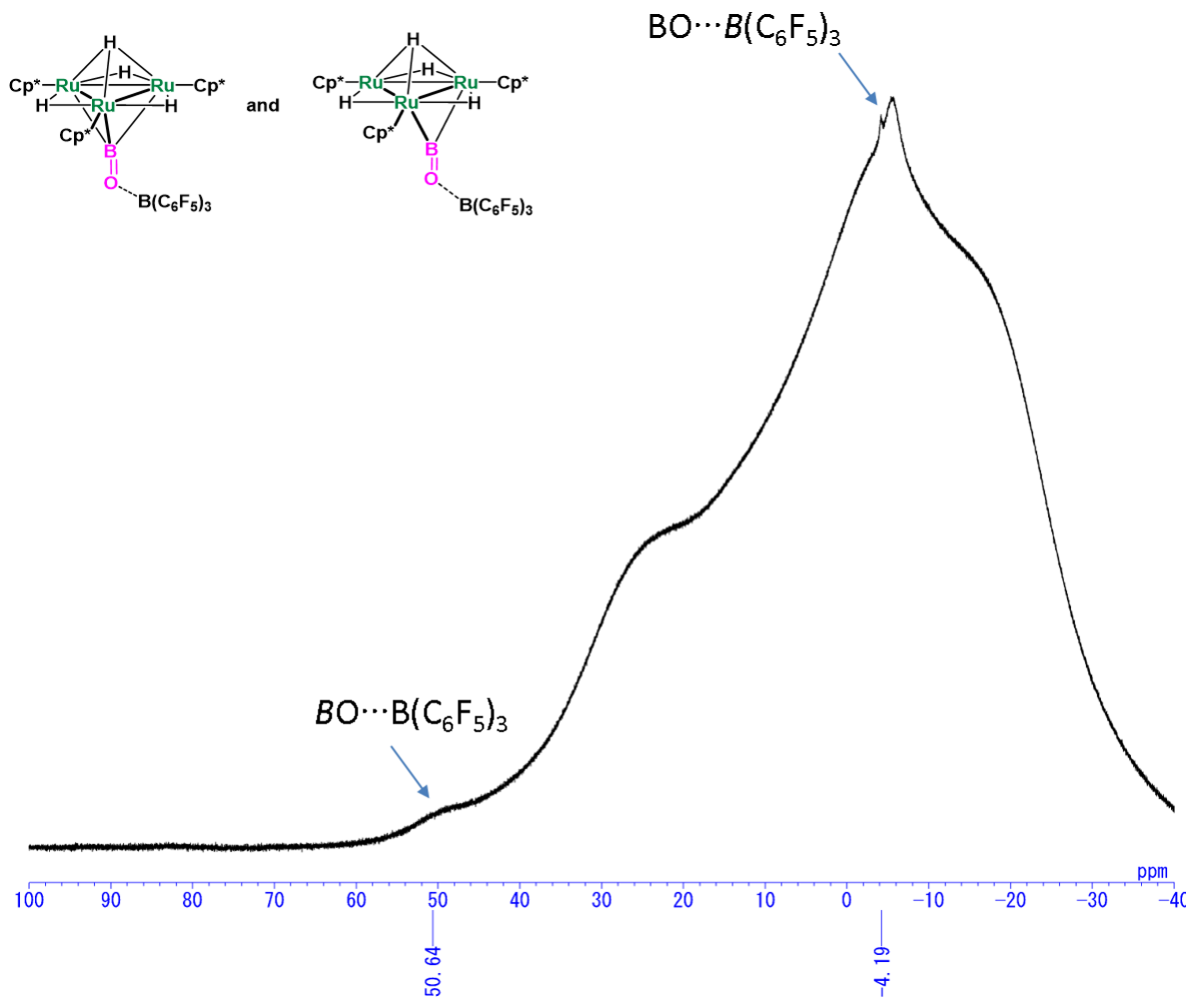

Figure S27. ${ }^{11} \mathrm{~B}\left\{{ }^{1} \mathrm{H}\right\}$ NMR spectrum of $5\left(129 \mathrm{MHz}, \mathrm{THF}-d_{8}, 25^{\circ} \mathrm{C}\right)$.

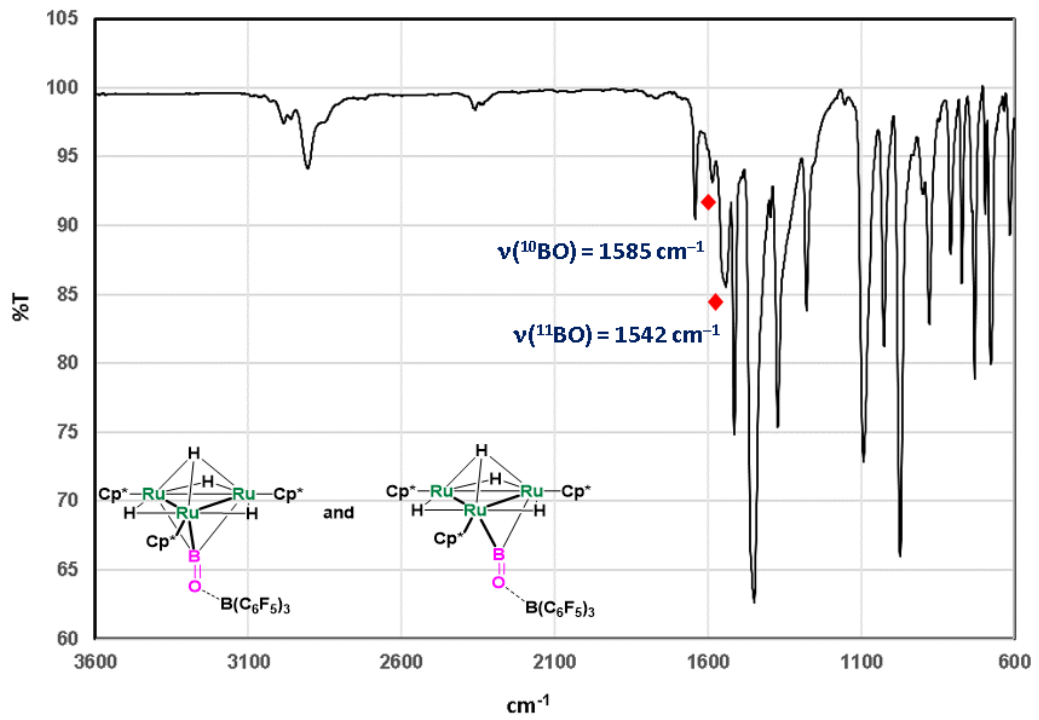

Figure S28. IR spectrum of $5\left(\mathrm{ATR}, \mathrm{cm}^{-1}\right)$. 


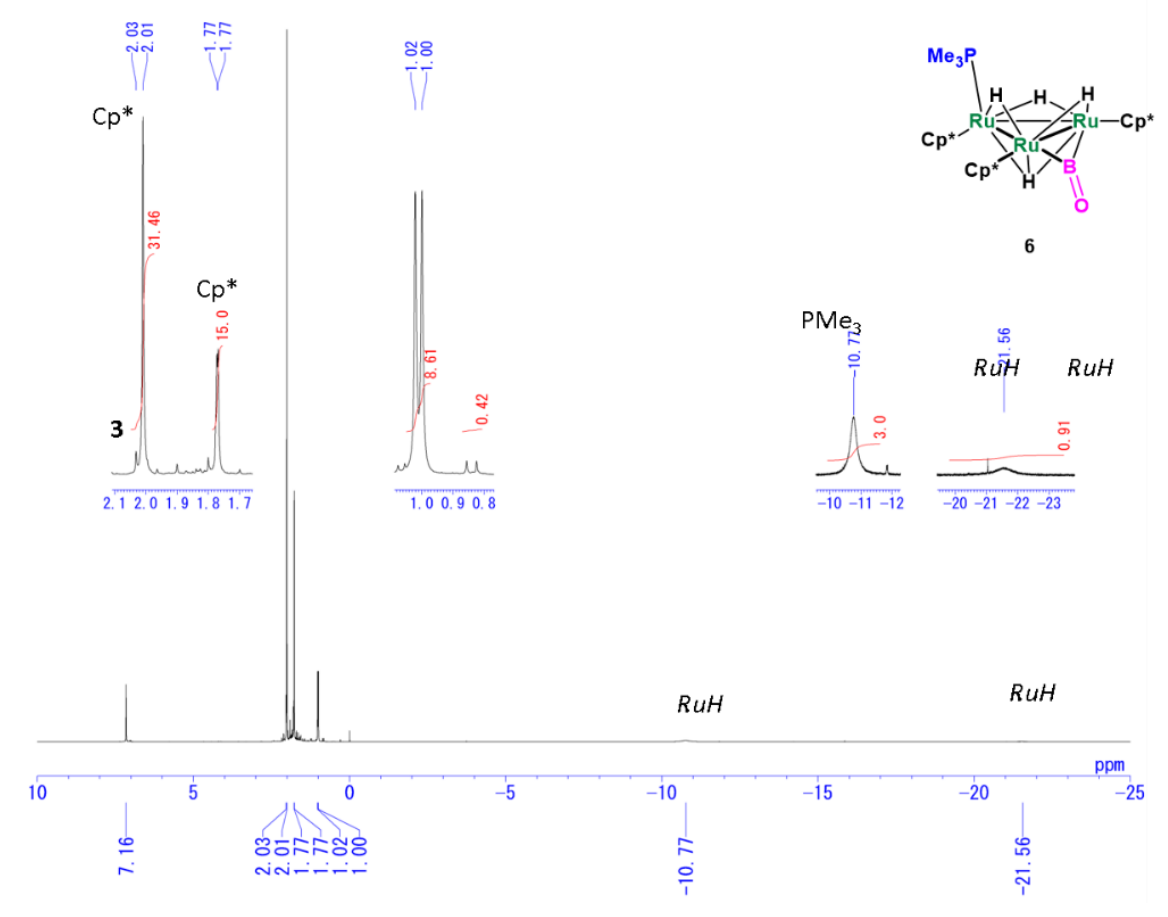

Figure S29. ${ }^{1} \mathrm{H}$ NMR spectrum of $6\left(400 \mathrm{MHz}, \mathrm{C}_{6} \mathrm{D}_{6}, 25^{\circ} \mathrm{C}\right)$.

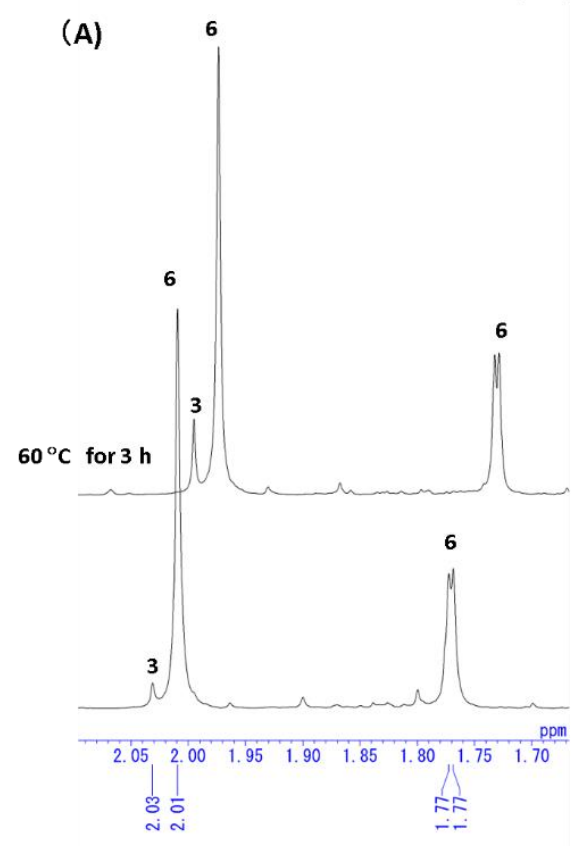

(B)

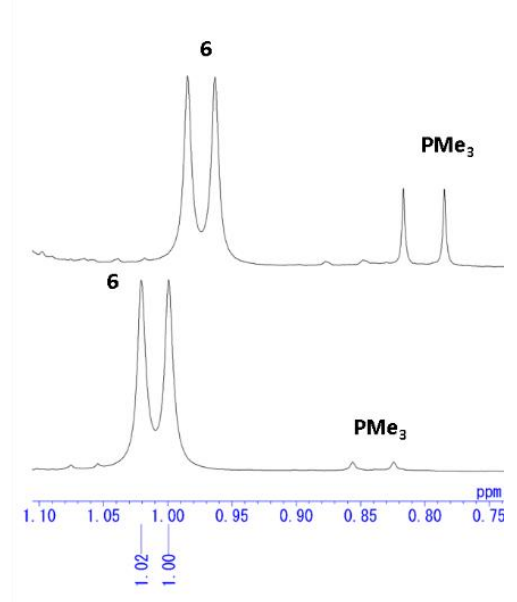

Figure S30. ${ }^{1} \mathrm{H}$ NMR spectra of 6 showing the (a) $\mathrm{Cp}^{*}$ and (b) $\mathrm{PMe}_{3}$ regions recorded before heating (below) and after heating at $60{ }^{\circ} \mathrm{C}$ for $3 \mathrm{~h}$ (above) $\left(400 \mathrm{MHz}, \mathrm{C}_{6} \mathrm{D}_{6}, 25^{\circ} \mathrm{C}\right)$. 


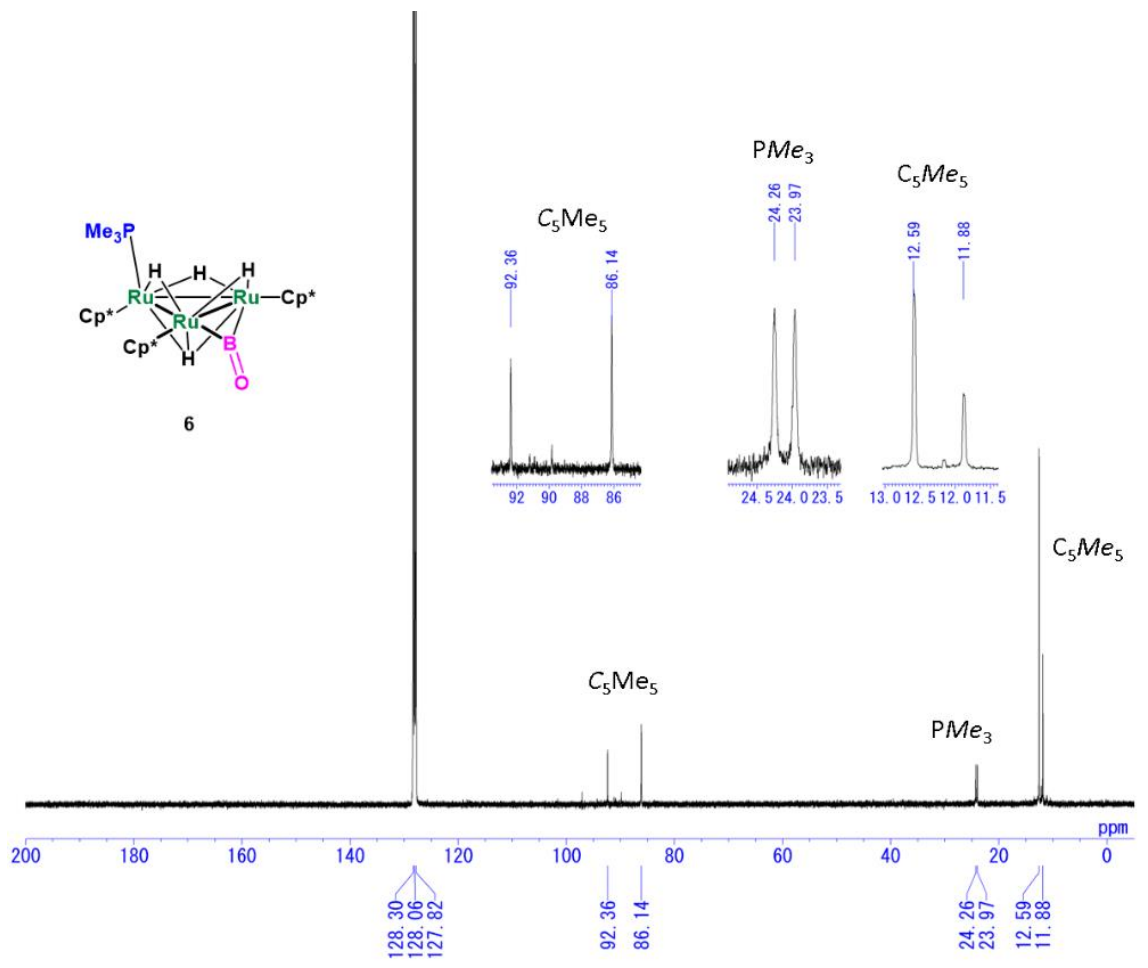

Figure S31. ${ }^{13} \mathrm{C}\left\{{ }^{1} \mathrm{H}\right\}$ NMR spectrum of $6\left(100 \mathrm{MHz}, \mathrm{C}_{6} \mathrm{D}_{6}, 25^{\circ} \mathrm{C}\right)$.

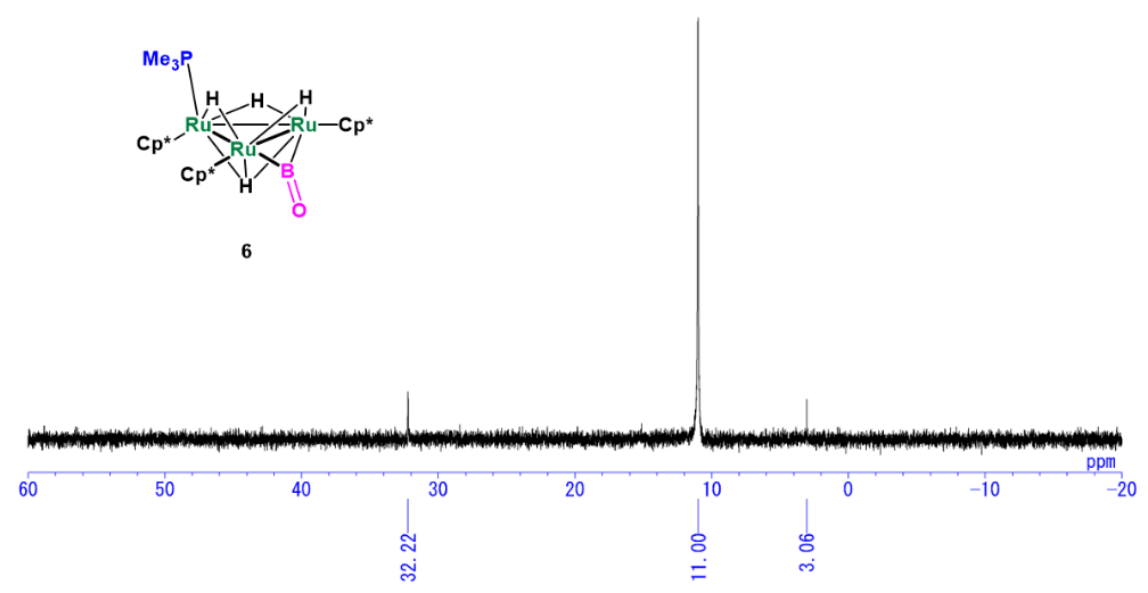

Figure S32. ${ }^{31} \mathrm{P}\left\{{ }^{1} \mathrm{H}\right\}$ NMR spectrum of $6\left(162 \mathrm{MHz}, \mathrm{C}_{6} \mathrm{D}_{6}, 25^{\circ} \mathrm{C}\right)$. 


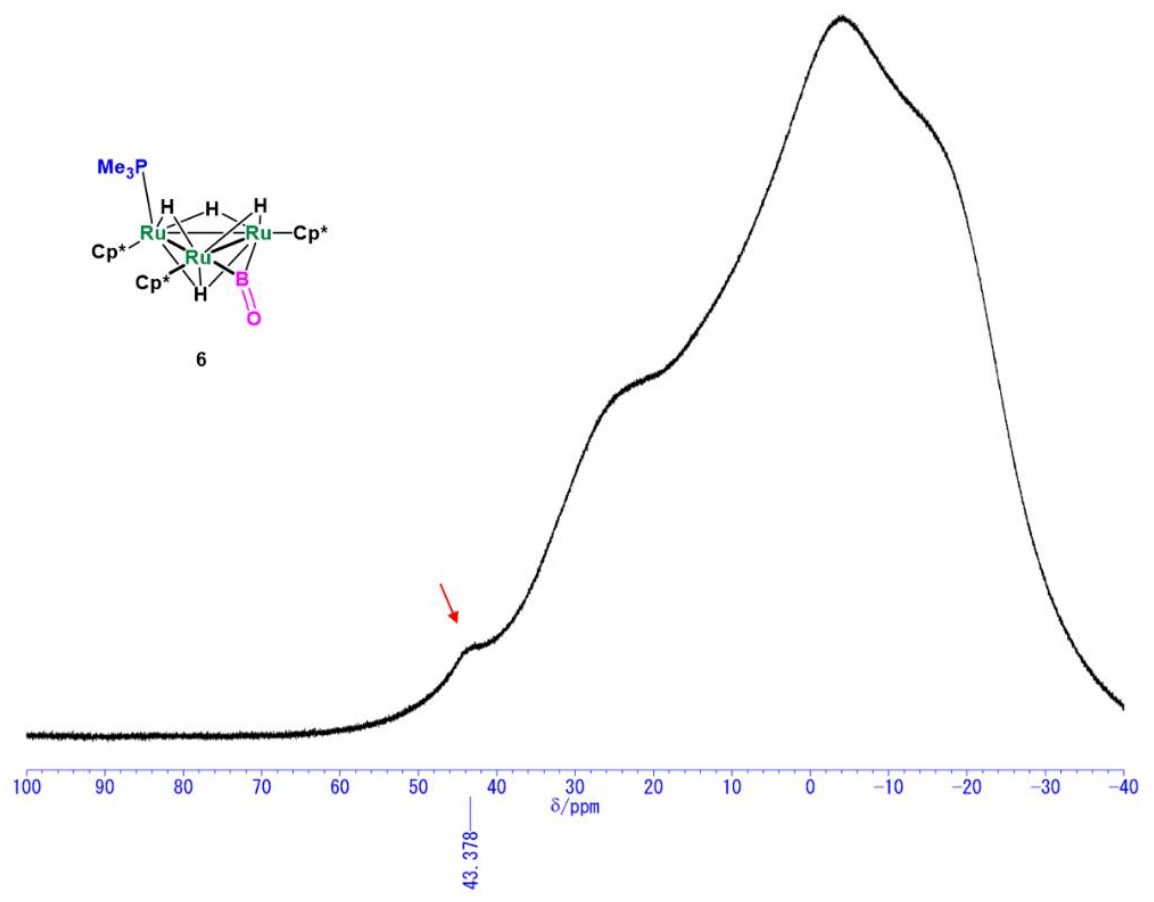

Figure S33. ${ }^{11} \mathrm{~B}\left\{{ }^{1} \mathrm{H}\right\}$ NMR spectrum of $6\left(129 \mathrm{MHz}, \mathrm{C}_{6} \mathrm{D}_{6}, 25^{\circ} \mathrm{C}\right)$.

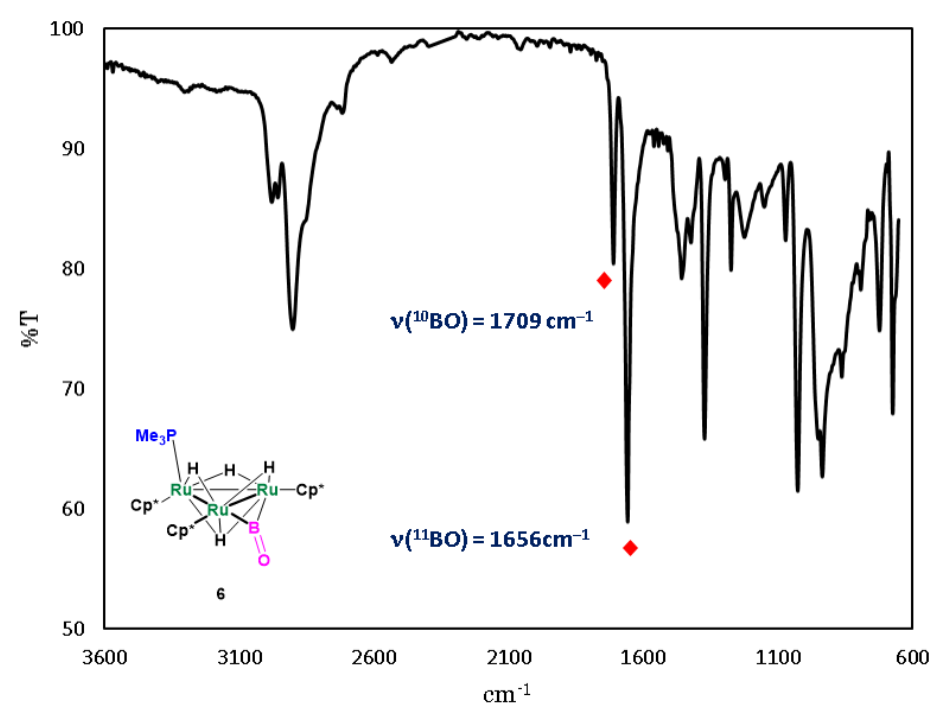

Figure S34. IR spectrum of $6\left(\mathrm{ATR}, \mathrm{cm}^{-1}\right)$. 


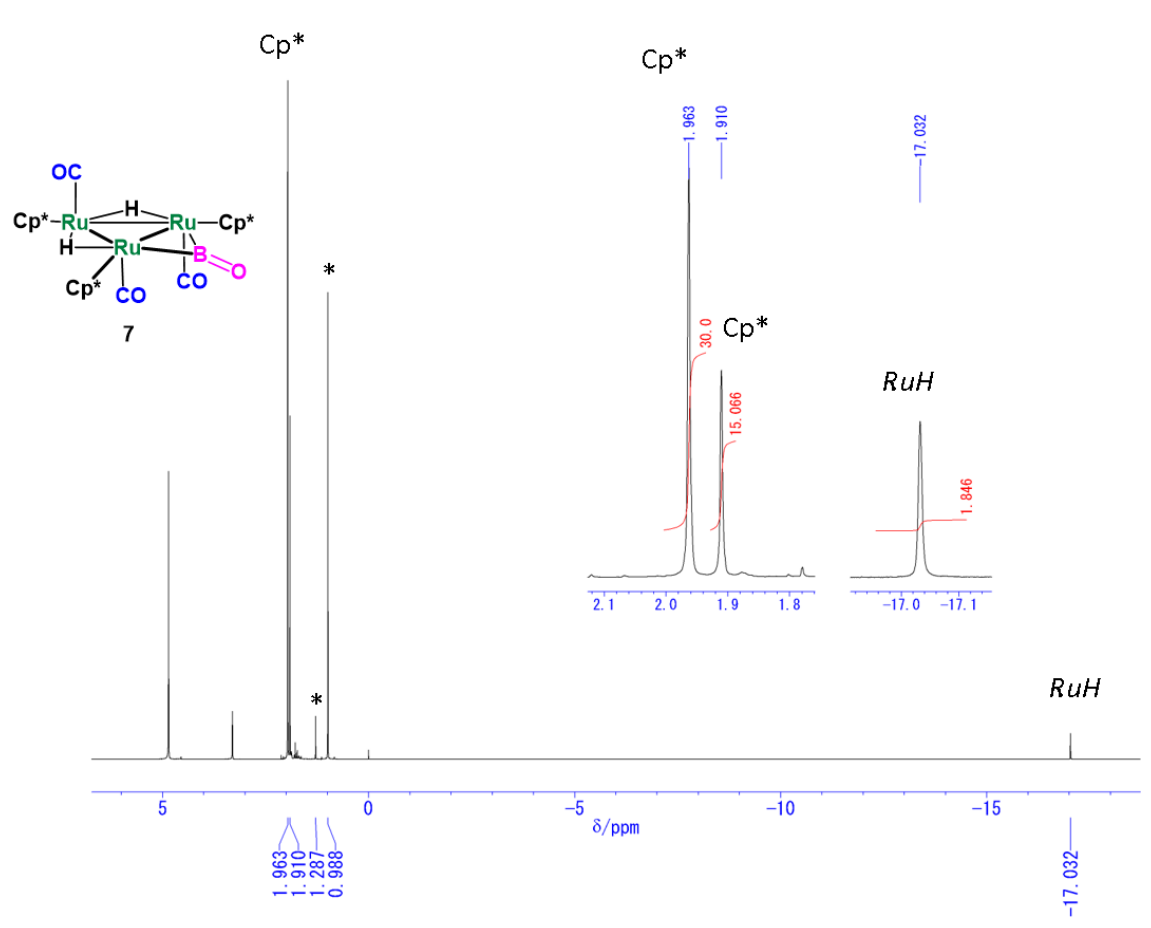

Figure S35. ' $\mathrm{H}$ NMR spectrum of 7. The signals with asterisks are derived from 2,2',4,4'tetramethylpentane used as an internal standard $\left(400 \mathrm{MHz}, \mathrm{CD}_{3} \mathrm{OD}, 25^{\circ} \mathrm{C}\right)$.

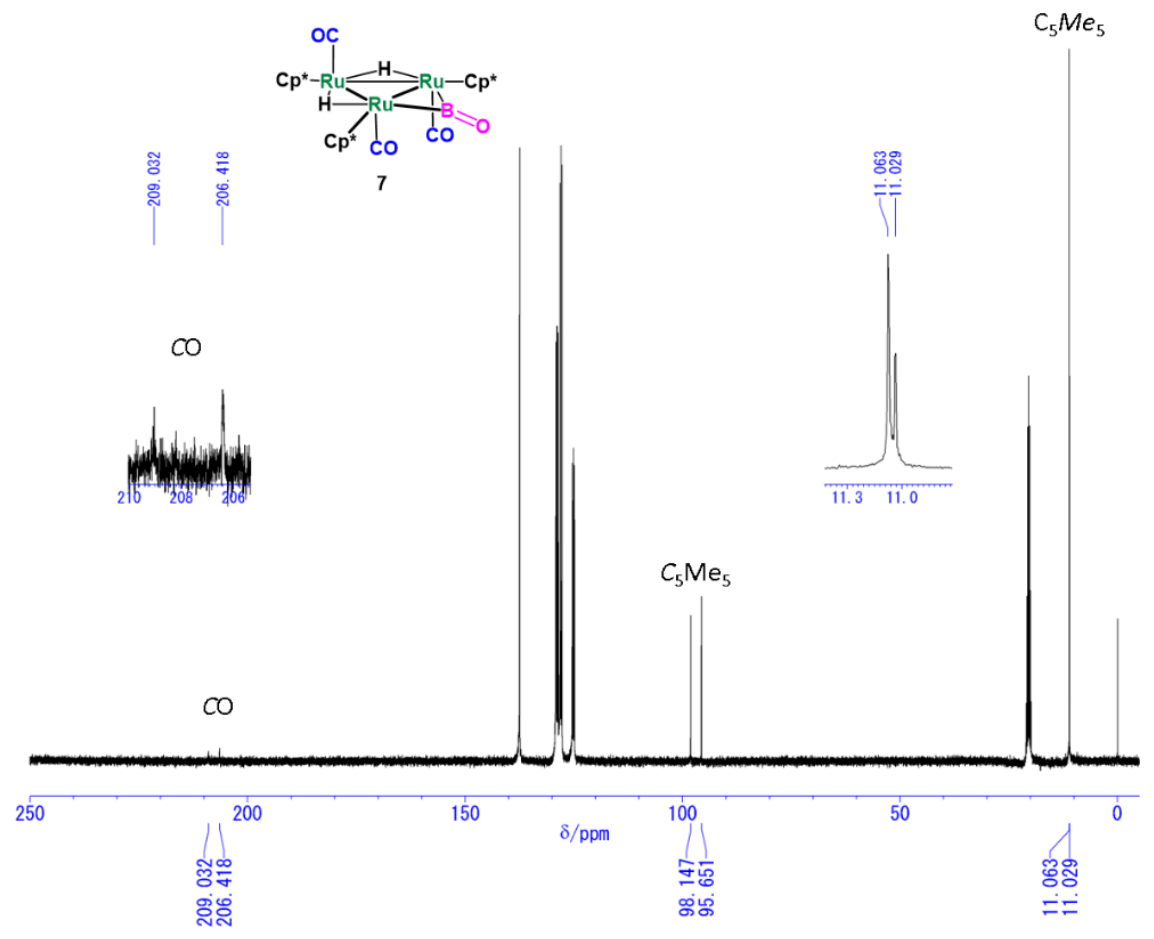

Figure S36. ${ }^{13} \mathrm{C}\left\{{ }^{1} \mathrm{H}\right\}$ NMR spectrum of $7\left(100 \mathrm{MHz}\right.$, toluene- $\left.d_{8}, 0{ }^{\circ} \mathrm{C}\right)$. 

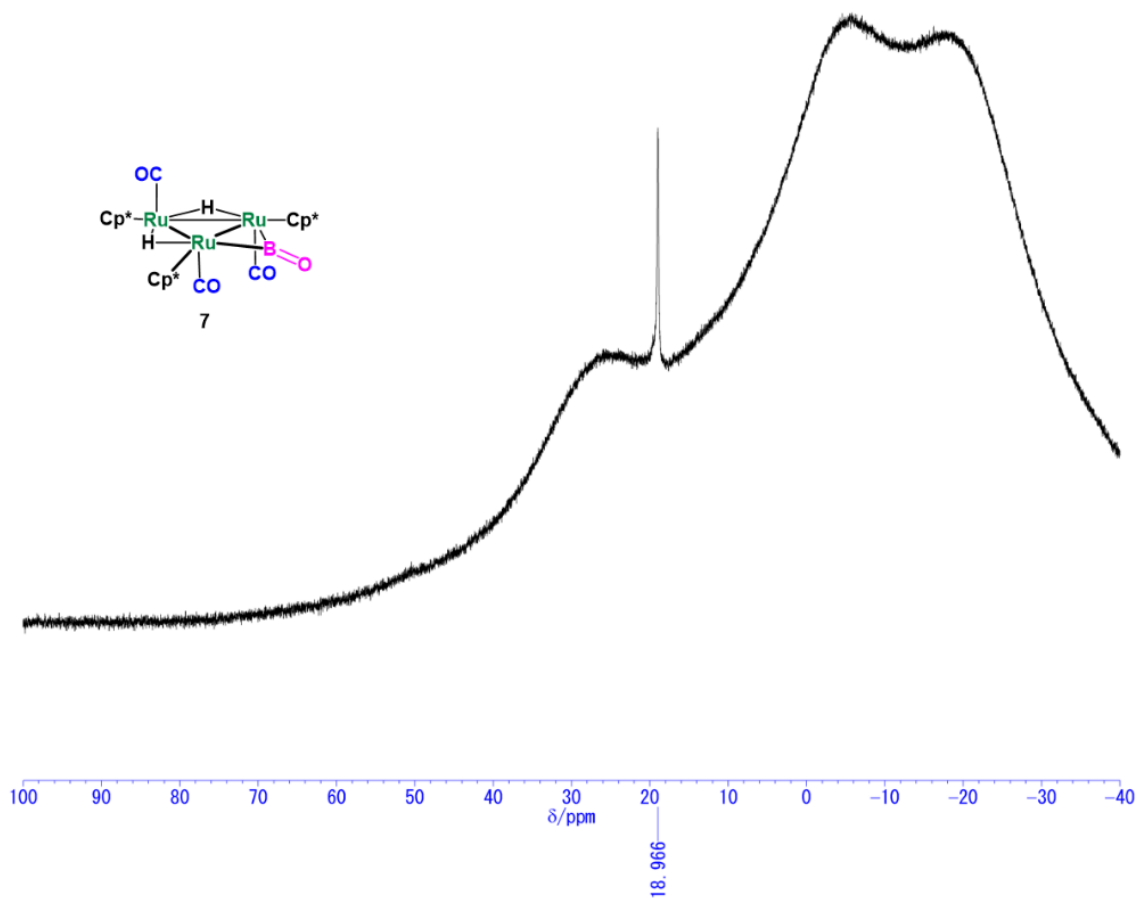

Figure S37. ${ }^{11} \mathrm{~B}\left\{{ }^{1} \mathrm{H}\right\}$ NMR spectrum of $\mathbf{3}\left(129 \mathrm{MHz}, \mathrm{CD}_{3} \mathrm{OD}, 25{ }^{\circ} \mathrm{C}\right)$.
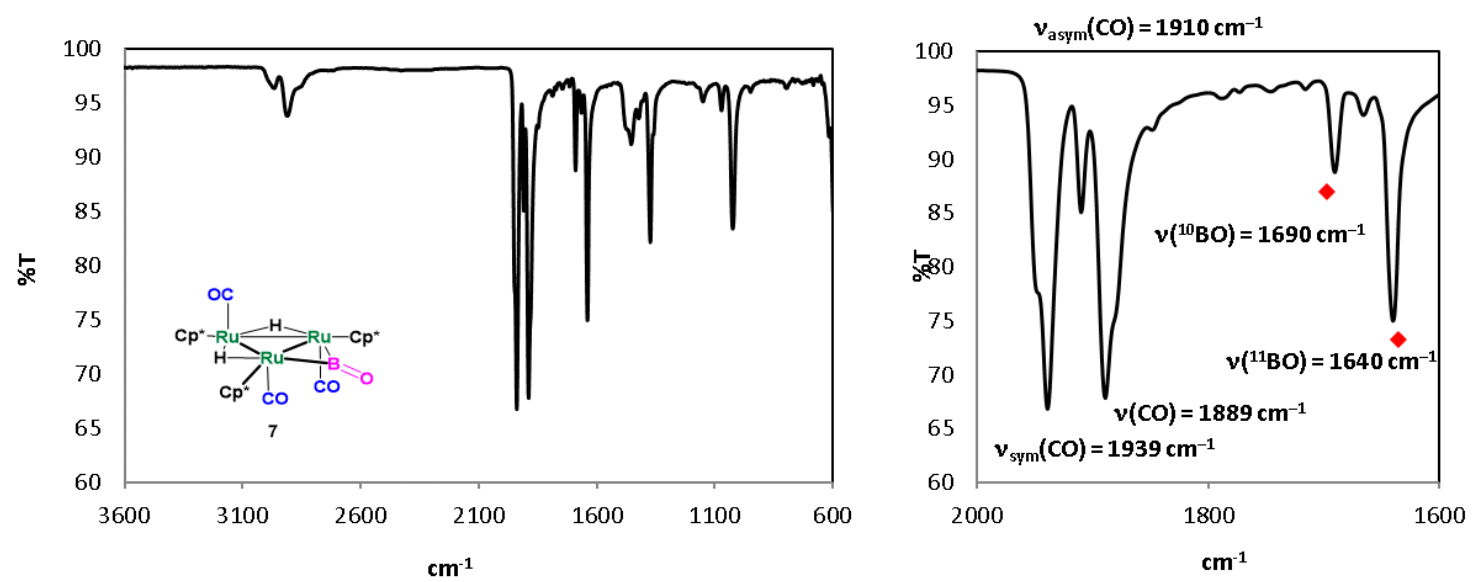

Figure S38. IR spectrum of 7 (ATR, $\left.\mathrm{cm}^{-1}\right)$. 


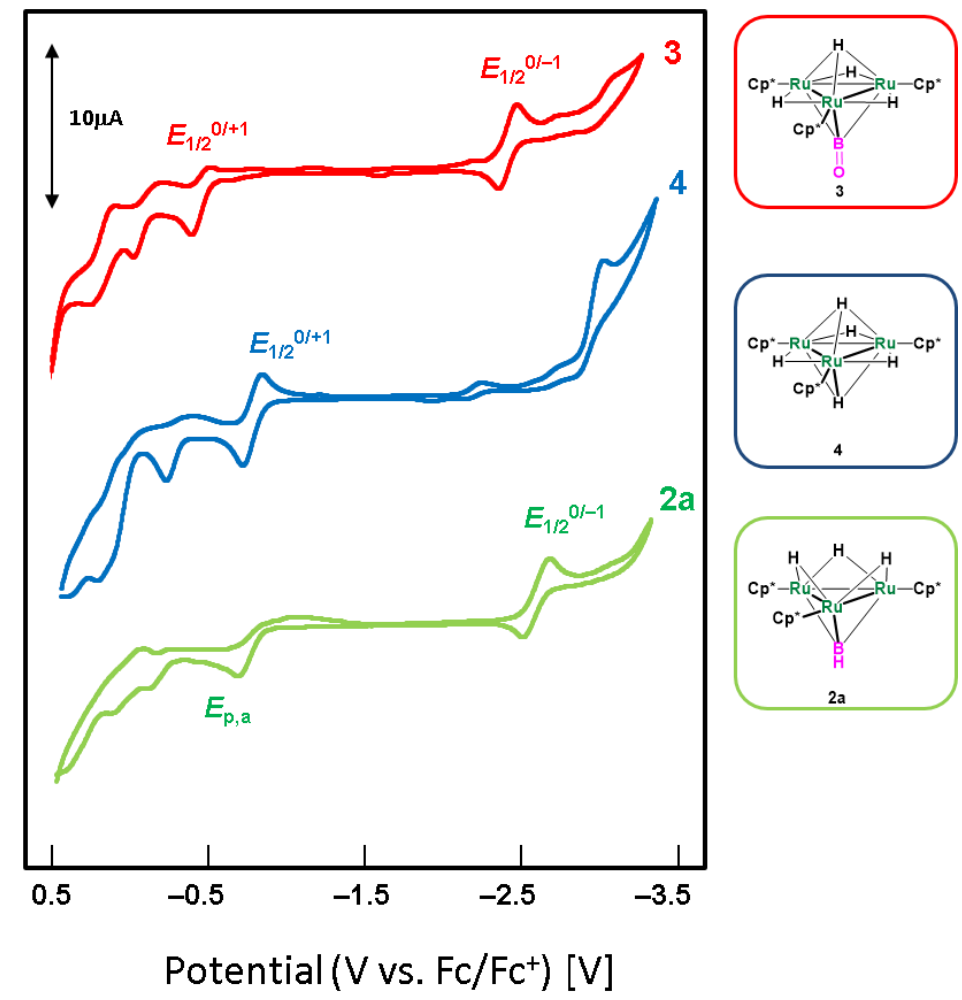

Figure S39. Cyclic voltammograms of 3, 4, and 2a measured in THF at $25^{\circ} \mathrm{C}$. Conditions: electrolyte, $0.1 \mathrm{M}\left[\mathrm{NBu}_{4}\right]\left(\mathrm{PF}_{6}\right)$; working electrode, $\mathrm{Pt}$; counter electrode, $\mathrm{Pt}$, scan rate $50 \mathrm{mV} / \mathrm{s}$. Potentials are referenced to $\mathrm{Cp}_{2} \mathrm{Fe} /\left[\mathrm{Cp}_{2} \mathrm{Fe}\right]^{+}$.

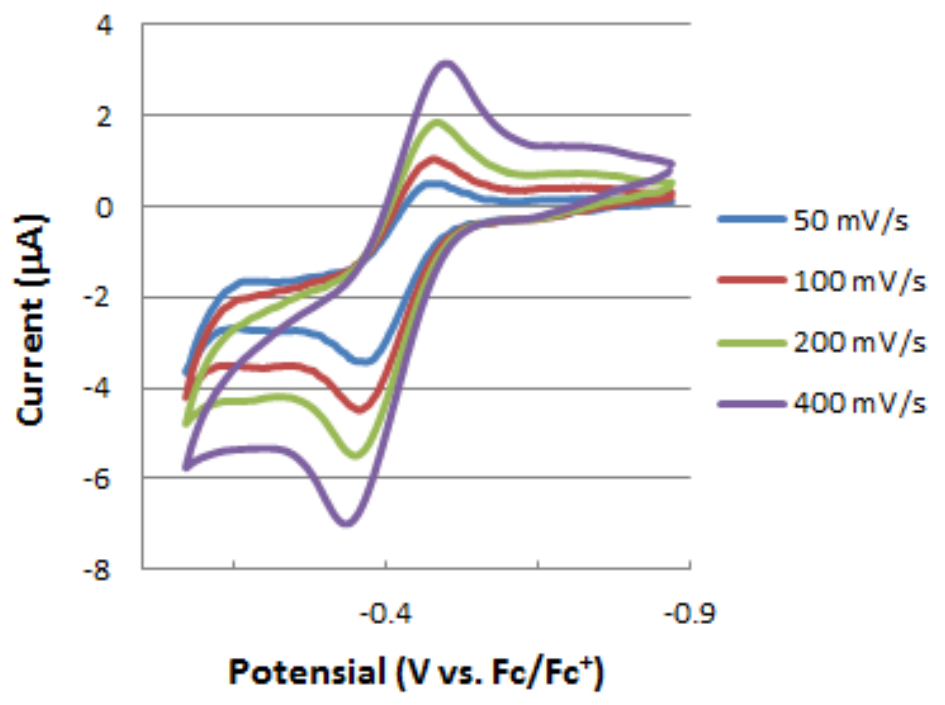

Figure S40. Cyclic voltammograms of $\mathbf{3}$ in THF at $25^{\circ} \mathrm{C}$ recorded at various scan rate. Conditions: electrolyte, $0.1 \mathrm{M}\left[\mathrm{NBu}_{4}\right]\left(\mathrm{PF}_{6}\right)$; working electrode, $\mathrm{Pt}$; counter electrode, $\mathrm{Pt}$; Scan range, from -0.87 to $-0.07 \mathrm{~V}$. Potentials are referenced to $\mathrm{Cp}_{2} \mathrm{Fe} /\left[\mathrm{Cp}_{2} \mathrm{Fe}\right]^{+}$. 